\title{
HDAC11 is a novel regulator of fatty acid oxidative metabolism in skeletal muscle
}

Erica Hurtado ${ }^{1 *}$, Yaiza Núñez-Álvarez ${ }^{1^{*}+}$ Mar Muñoz ${ }^{1}$, Cristina Gutiérrez-Caballero ${ }^{2}$, Josefina Casas ${ }^{3,4}$, Alberto M. Pendás ${ }^{2}$, Miguel A. Peinado ${ }^{1}$ and Mònica Suelves ${ }^{1 * *}$

*Equal contribution

${ }^{1}$ Program of Predictive and Personalized Medicine of Cancer, Germans Trias i Pujol Research Institute, Can Ruti Campus, Badalona, Spain. ${ }^{2}$ Institute of Cellular and Molecular Biology of Cancer, Salamanca, Spain. ${ }^{3}$ Institute of Advanced Chemistry of Catalonia, Barcelona (IQAC-CSIC), Spain. ${ }^{4}$ Liver and Digestive Diseases Networking Biomedical Research Centre, Madrid, Spain

Erica Hurtado: ehurtado@igtp.cat

Yaiza Núñez-Álvarez: yaiza.nunez-alvarez@igh.cnrs.fr

Mar Muñoz: $\underline{\text { mmunoz@igtp.cat }}$

Cristina Gutiérrez-Caballero: cristina@exonate.com

Josefina Casas: fina.casas@iqac.csic.es

Alberto M. Pendás: amp@usal.es

Miguel A. Peinado: mpeinado@igtp.cat

Mònica Suelves: msuelves@igtp.cat

+Present address: Institute of Human Genetics, IGH-UMR9002 CNRS/UM, 34396 Montpellier Cedex 5, France

** Correspondence to:

Mònica Suelves

Germans Trias i Pujol Research Institute (IGTP), Program of Predictive and Personalized Medicine of Cancer, Can Ruti Campus, Crta de Can Ruti, Camí de les Escoles s/n, 08916 Badalona, Spain

Phone: 34935543058

E-mail: msuelves@igtp.cat 


\section{Abstract}

Skeletal muscle is a highly metabolic tissue that requires efficient carbohydrates and fatty acids oxidative metabolism to meet its energy demands. HDAC11 is highly expressed in muscle tissue, but its role in skeletal muscle physiology has never been investigated. Here, we describe for the first time the consequences of HDAC11 genetic deficiency in skeletal muscle, which results in the improvement of muscle function enhancing fatigue resistance and muscle strength. Loss of HDAC11 had no obvious impact on skeletal muscle structure, but increased the number of oxidative myofibers by promoting a glycolytic-to-oxidative muscle fiber switch. Unexpectedly, HDAC11 was found to localize in mitochondria and its deficiency enhanced mitochondrial content and increased fatty acid $\beta$-oxidation through activating the AMPK-ACC signaling pathway, thus providing a mechanistic explanation for the improved muscle strength and fatigue resistance. Overall, our data reveal a new and unique role of HDAC11 in the maintenance of muscle fiber-type balance by regulating mitochondrial lipid oxidation. These findings shed light on the mechanisms governing muscle metabolism and may have implications for chronic muscle metabolic disease menagement.

Keywords: HDAC11, skeletal muscle, fiber type, mitochondria, oxidative metabolism, acylcarnitines, fatty acid oxidation, fatigue resistance

Abbreviations: ACC, acetyl-CoA carboxylase; AMPK, AMP activated protein kinase; COX IV, cytochrome c oxidase subunit IV; CPT1, carnitine O- 
palmitoyltransferase 1; CSA, cross-sectional area; EDL, extensor digitorum longus; FAO, fatty acid oxidation; GC, gastrocnemius; HDAC11, histone deacetlyase 11; $\mathrm{H} / \mathrm{E}$, hematoxylin and eosin; KO, knockout; mtDNA, mitochondrial DNA; MYHC, myosine heavy chain; PGC1 $\alpha$, peroxisome proliferator-activated receptor $\gamma$ coactivator; PL, plantaris; RT-qPCR, reverse transcription quantitative polymerase chain reaction; SL, soleus; TA, tibialis anterior; TFAM, mitochondrial transcription factor A; TFB2M, transcription factor B2 of the mitochondria; TOM20, translocase of outer mitochondrial membrane 20; WB, Western Blot; WT, wild type. 


\section{Introduction}

Skeletal muscle is the largest tissue in mammalian organisms and consists of heterogeneous muscle fibers (myofibers) with distinct functional characteristics which are classified based on contractile speed, myosine heavy chain (MHC) expression, metabolic capacity and myoglobin content [1]. Slow-twitch fibers (also called type I myofibers) express myosin heavy chain I (Myh7 gene), display oxidative metabolism, are rich in mitochondria and myoglobin content and show high endurance capacity. In contrast, fast-twitch myofibers are characterized by a fast contraction time and show reduced mitochondrial density associated with a predominant glycolytic metabolism. Fast-twitch myofibers are sub-classified in the oxidative type IIA (expressing Myh2 gene), the intermediate type IIX (expressing Myh1 gene) and the glycolytic type IIB (expressing Myh4 gene), being this last fiber type the fastest one [1]. Different skeletal muscles contain variable proportions of slow/fast myofibers, which determines their functional properties in terms of metabolic capacity, contraction speed and physical performance (endurance and strength). In addition, muscle fiber type composition may be important in pathological contexts, as reported in certain chronic muscular diseases. For instance, fast-twitch myofibers are more susceptible to suffer degenerative changes in Duchenne muscular dystrophy (DMD) patients than slow-twitch myofibers, and reduced amount of oxidative fibers may contribute to insulin resistance and increase risk of obesity [2]. Therefore, the switching of skeletal muscle fiber type from fasttwitch to slow-twitch affects the maintenance of energy homeostasis, muscle contraction capacity and fatigue resistance. 
Histone deacetylase 11 (HDAC11) is the only member of the class IV HDAC subfamily and is the latest HDAC protein identified [3]. HDAC11 is expressed in specific tissues, such as brain, skeletal muscle, heart, testis and kidney [3]. HDAC enzymes catalyse the removal of acetyl groups from acetylated lysine residues, in histones and non-histone proteins, and are implicated in several biological processes including development, inflammation and metabolism [4-6]. Very recently, HDAC11 was reported to have a very potent defatty-acylase activity compared to its minor deacetylase activity $[7,8]$ and the mitochondrial enzyme SHMT2 (serine hydroxymethyltransferase 2) was biochemically validated as the first HDAC11 defatty-acylated substrate [9]. Interestingly, loss of HDAC11 was recently shown to promote thermogenic capacity, stimulate brown adipose tissue formation and, in response to high-fat feeding attenuate obesity and reduce metabolic syndrome $[10,11]$.

Here we address for the first time the role of HDAC11 in skeletal muscle. Our results show that genetic loss of HDAC11 has no obvious impact on skeletal muscle neither structure nor muscle growth. However, lack of HDAC11 promotes a glycolytic-tooxidative muscle fiber switch, increases mitochondrial content and enhances the oxidation of fatty acids in muscle tissue. Importantly, depletion of HDAC11 confers muscle increasing fatigue resistance and strength. These findings uncover HDAC11 as a crucial regulator of mitochondrial lipid oxidation in skeletal muscle tissue, with potentially important implications for metabolic muscle diseases. 


\section{Results}

HDAC11 knockout mice display normal skeletal muscle growth, morphology and integrity

HDAC11 is expressed in a tissue-specific manner, being the highest expression in brain followed by skeletal and cardiac muscles (Fig 1A). To study the biological function of HDAC11 in vivo, we generated HDAC11 total knockout mice (HDAC11 ${ }^{-1}$ or KO mice) by a targeted depletion of floxed exon 3 of the Hdac11 gene (Supplemental Fig 1A). The correct genotyping of wild type (WT) and KO mice was assessed by PCR, using mouse-tail genomic DNA (Fig 1B). Loss of HDAC11 protein in $\mathrm{HDAC} 11^{-1}$ mice was confirmed by Western blot analysis of muscle protein extracts and compared with WT (Supplemental Fig 1B). HDAC1 $11^{-/}$mice were born at a normal Mendelian ratio and were indistinguishable from WT counterparts (Fig 1B and Supplemental Fig 1C). To analyze whether the lack of HDAC11 could affect body mass with age, we weighted WT and KO mice at neonatal (10 days), weaning (21 days), adult (90 and 180 days) and old (360 days) age, but no major differences were noticed between genotypes (Fig 1B). Comparison of different skeletal muscles (soleus, extensor digitorum longus (EDL), plantaris, tibialis anterior (TA) and gastrocnemius) showed similar appearance and weights in WT and KO adult mice (Fig 1C). To assess the consequences of HDAC11 deficiency on skeletal muscle structure, we quantified the number and cross-sectional area (CSA) of myofibers in hematoxylin and eosin $(\mathrm{H} / \mathrm{E})$-stained cryosections of adult muscles of both sexes. Figure 1D shows representative images from TA and soleus muscles from control and $\mathrm{HDAC}_{1} 1^{-\kappa}$ mice. The quantification of the CSA of myofibers at the TA and 
soleus mid-belly showed no differences in the number of myofibers per muscle area or in fiber size, indicating normal morphology of HDAC11-deficient skeletal muscles, both in male and females. Collectively, these results showed that lack of HDAC11 does not affect neither skeletal muscle growth nor the maintenance of skeletal muscle mass, integrity and physiological structure.

\section{HDAC11-deficiency induces a glycolytic-to-oxidative muscle fiber switch in} vivo

HDAC11 is highly expressed in skeletal muscle tissue, but its relative abundance in slow/oxidative versus fast/glycolytic skeletal muscles was not known. Comparison of slow myofiber-enriched soleus muscle to fast myofiber-enriched TA and EDL muscles by RT-qPCR showed that HDAC11 mRNA was expressed over six folds higher in fast TA and EDL muscles (Fig 2A). Higher levels of HDAC11 protein were also detected by Western blot (WB) in TA compared with soleus muscles (Fig 2B), suggesting a predominant role of HDAC11 in fast-twitch muscles.

To assess whether HDAC11 deficiency could alter myofiber composition in skeletal muscles, we first compared the myosin protein levels, using specific antibodies for MyHC-IIB and MyHC-IIA/IIX isoforms, in WT and HDAC11-deficient muscles. The results clearly showed a reduction of the fastest MyHC-IIB isoform and an increase of $\mathrm{MyHC}-\mathrm{IIA} / \mathrm{IIX}$ in TA KO muscles (Fig 2C). Next, we performed immunohistochemistry analysis on frozen cryo-sections to identify and quantify the

number of type IIA, IIX and IIB myofibers. As shown in Figure 2D, we found a significant increase in the number of IIA myofibers and a concomitant decrease in 
the number of IIX myofibers in HDAC11-deficient muscles. Thus, HDAC11 is more expressed in fast-twitch skeletal muscles and its absence results in a myofiber shift towards a slower/oxidative phenotype.

\section{HDAC11-deficiency does not alter the expression levels of slow- and fast- related muscle genes}

Histone deacetylases are epigenetic regulators that can modulate gene expression. To address whether HDAC11 could be a transcriptional regulator of slow-twitch program, we analyzed the expression levels of several slow-related genes in HDAC11-deficient and WT skeletal muscles, without detecting major differences between genotypes. By RT-qPCR (Fig S2) we compared the expression levels of the transcription factors Mef2c, Mef2d, Nfatc1 and Prox1 essential for slow fiber-type determination [12-15], the slow-type sarcomeric proteins Tnni1 (troponin 11) and Tnnc1 (troponin C1) and the calcium regulator Serca2 (sarcoendoplasmic reticulum calcium transport ATPase). In addition, the expression levels of fast-type related genes did not show differences between samples either. We analysed the expression of the transcription factor Six 1 , the fast-type sarcomeric proteins Tnni2 (troponin I2) and Tnnc2 (troponin C2) and the fast-related calcium regulator Serca1 and SIn (Sarcolipin). Altogether, these results suggest that HDAC11 is not a major transcriptional regulator of fast/slow transcriptional programs.

Little is known about the neurological functions of HDAC11, although it is highly expressed in neurons and oligodendrocytes [16]. We addressed whether muscle innervation could be altered by HDAC11 deficiency by comparing the expression 
levels of synapse-specific genes, including muscle-specific AchR subunits (Chrn $\alpha$, $\beta, \chi$ and $\varepsilon$ genes), agrin, neuregulin, and myogenin. This initial study showed no differences in the expression of all these genes between genotypes (Fig. S2B), suggesting that synaptic transmission at the neuromuscular junctions may not be affected in the absence of HDAC11.

\section{HDAC11 is located in mitochondrion and its deficiency increases mitochondrial content}

It is well established that oxidative myofibers show both higher levels of myoglobin (the oxygen storing protein in muscle cells) and mitochondria [1]. To better characterize the observed glycolytic-to-oxidative switch in muscle of HDAC11deficient muscle, we analyzed myoglobin expression and the levels of PGC1 $\alpha$, a major regulator of mitochondrial biogenesis [16]. As shown in Figure $3 \mathrm{~A}$, the myoglobin expression level was significantly higher in HDAC11-deficient muscles, while PGC1 $\alpha$ expression remained unchanged (Fig 3B). To analyze mitochondrial content, we measured mitochondrial DNA levels (mt-DNA) in WT and HDAC11 ${ }^{-1-}$ muscles. mt-DNA (Cox2 and a mitochondrial DNA fragment named $f$-mtDNA) relative to genomic DNA (Sdha and RplpO genes) showed a high increase in mtDNA in HDAC11 $1^{--}$muscles (Fig 3C and Supplemental Fig 3). Next, we quantified the levels of mtTFA (TFAM) and mtTFB2 (TFB2M) as mitochondrial transcription factors involved in mtDNA replication and transcription and those of mitochondrial membrane proteins TOM20 and COX IV. As shown in Figure 3D and Supplemental 
Figure 3 , the expression levels of the four mitochondrial markers were higher in HDAC11 $1{ }^{--}$than WT muscles, suggesting that HDAC11-deficient skeletal muscles have higher mitochondrial content that could enhance their oxidative capacity.

Previous studies have located HDAC11 in the nucleus [3] or in both the nucleus and cytoplasm of mammalian cells $[17,18]$. Given the potential impact of HDAC11 in mitochondrial mass, we analyzed the HDAC11 subcellular localization in muscle cells. Through protein subcellular fractionation, HDAC11 was found to be located in mitochondria, as well as in cytoplasm and nucleus (Fig 3E). This result places HDAC11 for the first time in mitochondria and suggests that HDAC11 could be a potential regulator of mitochondrial function and oxidative metabolism in skeletal muscle tissue.

\section{HDAC11 deficiency enhances the activation of the AMPK-ACC signaling pathway}

To address whether a more oxidative muscle metabolism occurs in the absence of HDAC11 we analyzed the phosphorylation level of the energy sensor AMPK in WT and $\mathrm{HDAC} 11^{-/-}$muscles. As shown in Figure 4A, lack of HDAC11 results in higher phosphorylation levels of AMPK in HDAC11-deficient muscles. CPT1 (carnitine palmitoyltransferase 1) is the enzyme that initiates the first step of the oxidative pathway in the mitochondrial matrix being a key regulator of mitochondrial longchain fatty acid $\beta$-oxidation [19]. Recently, loss of HDAC11 was shown to elevate CPT1 activity in liver tissue thus enhancing metabolic activity [10]. AMPK signaling is involved in lipid metabolism through phosphorylation of acetyl-CoA carboxylase 
(ACC), which upon being phosphorylated removes CPT1 inhibition and allow fatty acid oxidation (FAO) [20,21]. Interestingly, as shown in Figure 4A, ACC phosphorylation was also significantly higher in HDAC11-deficient muscles, suggesting an increase in CPT1 activity that could result in a higher FAO capacity in muscles lacking HDAC11.

\section{Fatty acid metabolism is more efficient in HDAC11- deficient muscles}

To address whether the CPT1-dependent fatty acid $\beta$-oxidation was altered in HDAC11-deficient samples, we quantified acylcarnitine levels in lipid extracts of WT and $\mathrm{HDAC} 11^{-/}$muscles by ultra-high performance liquid chromatography time-offlight mass spectrometry (UHPLC-TOF-MS). As show in Figure 4B, the total amount of acylcarnitines was reduced by more than half in HDAC11-deficient muscles suggesting a more efficient fatty acid oxidation in $\mathrm{HDAC} 11^{-/}$mice.

To assess whether the reduction of acylcarnitines levels in the absence of HDAC11 was due to an increase of fatty acid $\beta$-oxidation, we compared FAO activity in WT and HDAC11 KO muscles by analyzing the oxidation of octanoyl-CoA. As shown in Figure $4 \mathrm{C}$, the oxidation of octanoyl-CoA to acetyl-CoA and the concomitant production of FADH2 and NADH in the mitochondria were significantly higher in $\mathrm{HDAC}_{11^{-/}}$muscles, indicating a more efficient FAO in HDAC11-deficient muscles. Altogether, these results indicate that HDAC11 plays an important role in the 
regulation of lipid metabolism through regulating the fatty acid oxidation capacity in skeletal muscle.

\section{HDAC11-deficient mice show better muscle performance}

Skeletal muscle is the tissue with higher energy expenditure and during prolonged exercise its metabolism becomes more oxidative, with a switch to higher fatty acid oxidation [22]. To test whether the increased FAO capacity observed in HDAC11deficient muscles correlates with higher muscle endurance, WT and HDAC11 KO mice were exposed to increased exercise load on a treadmill machine, equipped with electronic shock grids, with progressive speed in a $20 \%$ slope and time until exhaustion was quantified. As shown in Figure 5A, HDAC11-deficient mice ran 25\% more time, at higher speed, and reached a $43 \%$ longer distance than WT mice. These results show an enhanced fatigue resistance in absence of HDAC11, consistently with the increased fatty acid oxidative capacity. Also the parameters of work and power, calculated based on body weight and slope, were significantly higher in HDAC11-deficient mice indicating an improved exercise capacity in KO mice (Fig 5A). In addition, using an automatic grip strength meter, we showed increased hind limb grip strength in HDAC11 KO mice compared with WT (Fig 5B). Finally, we performed the four limbs hang test (also known as Kondziella's inverted grid test), which uses a wire grid system to measure the time that mice can sustain limb tension to oppose their gravitational force and weight. HDAC11-deficient mice performed better, as shown by the longer time they sustained tension compared to WT all the days that the experiment was repeated (Fig 5C). Altogether, these results 
showed that HDAC11 deficiency increases skeletal muscle endurance and strength resulting in an improvement of muscle performance. 


\section{Discussion}

Our study uncovers a new role of HDAC11 in mitochondrial lipid oxidation and fibertype composition balance in skeletal muscle. HDAC11 is the latest HDAC protein identified and it is the smallest protein of the HDAC family, with the peculiarity that $80 \%$ of the protein sequence corresponds to the catalytic domain [3]. HDAC11 contains the two active site residues present in all eukaryotic HDAC proteins [3]. However, given the differences in the catalytic core region between HDAC11 and the rest of HDACs, HDAC11 established a new class of HDACs, class IV, being its unique member at present $[3,23]$. HDAC11 is highly expressed in brain, skeletal muscle, heart, kidney, testis and hematopoietic cells, but its biological functions are largely unknown. Here, we evaluate for the first time the consequences of HDAC11 deficiency in skeletal muscle tissue.

HDAC11-deficient skeletal muscles are morphologically indistinguishable from WT ones, showing very similar weight, growth rate and myofiber number and crosssectional area, both in adult males and females. These results indicate that HDAC11 is dispensable for skeletal muscle development, growth and establishment of tissue structure. However, skeletal muscle is composed by distinct myofiber types that differ in contractile and metabolic properties, and the proportion of slow/oxidative and fast /glycolytic myofibers in the distinct skeletal muscles determines their functional properties [24]. Interestingly, HDAC11 is more expressed in fast/glycolytic than in slow/oxidative muscles and its unique deficiency results in a shift towards a more oxidative phenotype. Other groups have reported the expression of classical 
HDACs in slow versus fast skeletal muscles showing that class I HDACs (HDAC1, 2 and 3) are expressed at similar levels, both at mRNA and protein level, in the distinct skeletal muscles [25]. In contrast, class Ila HDACs (HDAC 4, 5, 7 and 9) mRNAs are more expressed in slow muscles, while class Ila HDAC proteins are more abundant in fast-twitch muscles, suggesting posttranscriptional mechanisms involved in their regulation [25]. Of interest, HDAC4 protein is more abundant in the slow soleus muscle, although the basis of this discrepancy is not known yet [26]. Notably, although class Ila HDACs proteins are more highly expressed in glycolytic muscles, its individual deficiencies do not alter muscle fiber-type composition, indicating a functional redundancy between them [25]. In contrast, soleus muscles from mutant mice with reduced number of HDAC lla alleles (HDAC5 ${ }^{-/-} \mathrm{HDAC}^{-/}{ }^{-}$, HDAC4 ${ }^{\mathrm{fl} /}$; Myo-

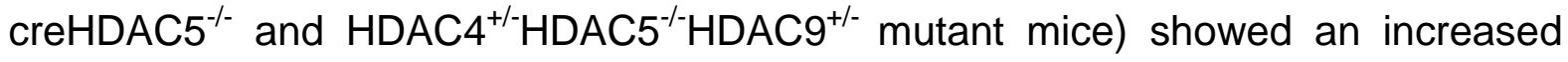
percentage of slow myofibers, suggesting that reducing the expression of class Ila HDACs below a threshold results in increased oxidative phenotype [25]. HDAC11 is the only member assigned to class IV HDACs and its deficiency does not modify the expression level of classes I and Ila HDACs in muscle tissue (data not shown). Importantly, lack of HDAC11 is sufficient to alter skeletal muscle fiber-type composition towards a slower/oxidative phenotype. This observation reveals a previously unknown role for HDAC11 in the maintenance of fiber-type balance in skeletal muscle (Fig 6).

It is well known that HDACs can interact with transcriptional activators and repressors to regulate gene expression of downstream genes [4]. For instance, class 
Ila HDACs repress the transcriptional activity of MEF2 transcription factors [27-30], which are important regulators of slow/oxidative fiber-type specific genes [12,31]. Our results show no changes in the expression levels neither of Mef2 nor of slow fiber- type specific genes such as Nfatc1, Prox1 and troponins Tnni1 and Tnnc1 genes, discarding HDAC11 as a transcriptional repressor of the slow-twitch gene program in skeletal muscle. In contrast, recent studies showed that HDAC11 functions as a repressor of the thermogenic gene program in brown adipose tissue through its association with the acetyl-histone-binding protein BRD2, on a specific enhancer that regulates Ucp1 expression [11]. HDACs are enzymes that catalyze the removal of acetyl and acyl groups from lysine residues on histone and nonhistone proteins [32]. HDACs do not bind directly to DNA but they are part of multiprotein complexes such as N-COR, SMART, NuRD and CoREST, which can localize to gene promoters and regulate their expression [17,33-36]. However, HDAC11 does not interact with high affinity with these classical interactors of classes I and Ila HDACs, while, surprisingly, HDAC11 seems to associate specifically with several members of the SMN complex responsible for spliceosome assembly [17]. Further experiments addressing the potential role of HDAC11 in mRNA splicing in skeletal muscle should be performed.

Previous studies showed that HDAC11 could localize in the nucleus and cytosol. In this latter compartment, HDAC11 physically interacts with HDAC6, which has predominant cytoplasm localization and regulates the acetylation levels of proteins such as tubulin, HSP90 and cortactin [37-39]. Importantly, our data demonstrate for the first time that HDAC11 is also located in mitochondria, suggesting that it may 
regulate mitochondrial biology. Consistent with this notion, our results showed increased mitochondrial DNA content and higher levels of TFAM, TOM20 and COX IV mitochondrial proteins in HDAC11-deficient skeletal muscles. This suggests higher mitochondrial mass in the absence of HDAC11, which is in agreement with the higher content of oxidative myofibers and increased myoglobin levels (Fig 6). PGC1 $\alpha$ is a central regulator of cellular metabolism and a master regulator of mitochondrial biogenesis [16]. Specific overexpression of PGC1 $\alpha$ in skeletal muscle results in the activation of oxidative genes and a fiber-type shift towards a more oxidative phenotype [40], although $\mathrm{PGC} 1 \alpha^{-/-}$mice contain apparently normal numbers of oxidative and glycolytic myofibers [41]. We found similar protein levels of PGC1 $\alpha$ in HDAC11-deficient and WT muscles, although we cannot rule out differences in its transcriptional activity as PGC1 $\alpha$ is heavily regulated at posttranscriptional level by phosphorylation, acetylation, methylation, glysolylation and ubiquitination [16].

Class III HDAC proteins, sirtuins (SIRT), are considered an independent HDAC subfamily because they require $\mathrm{NAD}^{+}$as a catalytic cofactor [32]. Sirtuin 3,4 and 5 are localized in mitochondria, and interestingly all of them can remove acyl groups, different from acetyl groups, in protein lysine residues. SIRT3 can remove 2hydroxyisobutyryl and crotonyl groups, SIRT4 can remove 3-hydroxy-3methylglutaryl and methylglutaryl groups and SIRT5 can remove glutaryl, malonyl and succinyl groups [42]. The cytoplasmatic sirtuins SIRT2 and SIRT6 are considered metabolic sensors and have also lysine defatty-acylase activity [43,44]. 
Intriguingly, very recent reports have demonstrated that HDAC11 has over 10.000fold more efficient defatty-acylase activity than the corresponding deacetylase activity, cleaving very efficiently long-chain acyl modifications on lysine residues, including the removal of myristoyl, palmitoyl and 3-hydroxydodecanoyl groups [7-9]. Furthermore, the metabolic enzyme SHMT2 (serine hydroxymethyltransferase), which is located mainly in the mitochondria and it is involved in one carbon metabolism, was recently identified as the first defatty-acylated substrate of HDAC11 in MCF-7 cells [45]. SHMT2 is a fatty-acylated protein whose fatty acyl groups can be removed by HDAC11 [9]. Interestingly, while the defatty acylation of SHMT2 does not affect SHMT2 enzymatic activity, SHMT2 colocalized with the lysosome marker LAMP1 in HDAC11 knock out cells, suggesting that lysine fatty-acylation in SHMT2 may promote lysosome translocation [9]. All these new data suggest that HDAC11 could be closer to sirtuins than to the zinc-dependent HDACs. Moreover, as the mitochondrial proteome is highly acylated [42], a critical role for HDAC11 in the removal of lysine acyl modifications in the mitochondria can be envisioned.

A recent proteomic and computational study in T cells performed by Cristea and coworkers identified the HDACs interactome, and proposed CPT1 as an HDAC11 interactor [17]. CPT1 is the mitochondrial enzyme responsible for the formation of acylcarnitines, the key regulatory enzyme of mitochondrial long-chain fatty acid- $\beta$ oxidation [20]. CPT1 activity is modulated by AMPK through ACC phosphorylation which reduces malonyl-CoA synthesis, the physiological inhibitor of CPT1 [20,21]. Our results showed higher phosphorylation levels of AMPK and ACC enzymes in 
HDAC11-deficient muscles, suggesting higher CPT1 activity. Currently, we do not know whether CPT1 is neither a fatty-acylated protein nor a direct target of HDAC11, but the lack of HDAC11 in skeletal muscle, and importantly also in liver, enhances CPT1 activity [10]. The quantification of acylcarnitine content in lipid extracts of WT and HDAC11-deficient muscles showed for the first time a significant reduction in the total amount of acylcarnitines in HDAC11-deficient samples, suggesting a more efficient fatty acid- $\beta$-oxidation in the absence of HDAC11. The measurement of FAO activity showed a significantly higher $\beta$-oxidation capacity in $\mathrm{HDAC} 11^{-/}$compared with WT skeletal muscles, indicating that HDAC11 is an important regulator of lipid metabolism in muscle tissue (Fig 6). Interestingly, very recently it has been reported that HDAC11-deficient mice are resistant to HFD-induced obesity, insulin resistance, and hepatic steatosis [10]. The authors showed that when HDAC11 __ mice were fed with HFD for 7 months, their body weight increase was significantly lower (around $25 \%$ less) compared to that of WT mice. In addition, plasma cholesterol, triglyceride, and insulin levels were also significantly reduced in HDAC11 KO mice, as well as the lipid accumulation in mice livers [10]. In that study, the authors demonstrated that the resistance of HDAC11 _ _ mice to (HFD)-induced obesity depended on metabolic mechanisms. Skeletal muscle constitutes over $40 \%$ of body mass in mammals and is a tissue with high metabolic demands, playing an important role in whole-body energy homeostasis. Obesity is often associated with increase fatty acid levels, which cause lipotoxicity, a common cause of insulin resistance [57]. Based on our results, we would suggest that is highly probable that the higher FAO 
capacity of muscle tissue significantly contributes to protect HDAC11-deficient mice from the HFD-induced obesity and metabolic syndrome.

Fatty acids are preferentially used during prolonged exercise as energy source, and the inability to use them causes exercise intolerance and muscle fatigue and weakness [58]. Importantly, our data demonstrated that HDAC11-deficient mice show enhanced muscle endurance and strength, suggesting that HDAC11 reduction or inactivation could benefit muscle function through this higher lipid oxidation capacity. The mechanisms underlying regulation of whole-body metabolism require the integration of several organs. HDAC11 is highly expressed in tissues with elevated metabolic rates, such as heart, kidney, liver, brain, adipose tissue, and skeletal muscle, contributing all of them to the body homeostatic balance. Intriguingly, Bagchi and coworkers published that HDAC11 total KO mice were healthy, but harbored excess of brown adipose tissue mass and showed increased thermogenesis and energy expenditure [11]. In our total KO mouse model, we demonstrate an improved skeletal muscle performance. Altogether, it is probable that total lack of HDAC11 may affect global energy metabolism, contributing to the described muscle phenotype. To dissect the HDAC11 functions in distinct cell types and understand its contribution to tissue metabolism, future studies with conditional KO mice should be performed.

Several human diseases are due to mitochondrial dysfunctions and alterations in fatty acid oxidation such as myosteatosis, with pathological accumulation of lipids in muscle tissue, which reduce its function and notably, it increases in aging and 
obesity [47]. Based on our results, it would be interesting to analyze whether the pharmacological inhibition of HDAC11 activity in animal models with myosteatosis could increase mitochondrial lipid oxidation and restore/improve muscle function.

In summary, the results presented here show that HDAC11 deficiency in skeletal muscle results in a shift toward a more oxidative phenotype by increasing mitochondrial lipid oxidation. We propose a previously unknown function of HDAC11 in mitochondria, as a new enzymatic remodeler of the mitochondrial lysine acylome regulating mitochondrial function. Future investigations are needed to identify HDAC11 defatty-acylated substrates and to dissect the molecular mechanisms between HDAC11 and metabolism. Interestingly, HDAC11 activity can be inhibited by new and selective drugs $[48,49]$, which could open avenues for the development of new therapies for the treatment of chronic muscle metabolic diseases. 


\section{Materials and methods}

\section{Animals}

HDAC11 total knockout mice (HDAC11 ${ }^{-/}$) were generated by crossing C57BL/6 mice carrying loxP sites flanking the exon 3 of Hdac11 gene (ENSMUSE00001222983, chr6: 91,109,155-91,109,255, mm9), with mice expressing CRE recombinase under the control of SOX2 promoter. The floxing of loxP sites causes a deletion of exon 3 leading to a premature stop codon in exon 4 (Supplemental Fig 1). That results in loss of function of HDAC11 by generating a truncated protein of 59 aa instead of 349 aa [50]. $\mathrm{HDAC} 11^{-}$animals were obtained from intercrossing heterozygote mice and WT littermates were used as controls. To identify the genotype by PCR reaction, primers flanking the deletion region were designed (forward primer: GGAAAGGCACTTTCACTTGC and reverse primer: ACCAACCACACAGCCCATA) and specific amplicon lengths were obtained (1104bp for WT and 181bp for HDAC $\left.11^{-/}\right)$. Mice were maintained in standard cages under standard conditions: constant temperature $\left(22^{\circ} \pm 2^{\circ}\right)$, relative humidity $(50 \pm 10 \%)$ and 12 -hour light/dark schedule. Standard rodent chow and clean water were available ad libitum. WT and ${\text { HDAC } 11^{-/}}^{-}$mice between 3-4 months of age were sacrificed by isoflurane inhalation followed by cervical dislocation, in accordance with the Guidelines laid down by the European Communities Council Directive of 24 November 1986 (86/609/EEC). At least 4 mice of the same sex per group were used in each experiment. 


\section{Histology and Immunohistochemistry}

Tibialis Anterior (TA) and Soleus (SL) muscles were dissected, embedded in OCT compound (ref. 00411243, VWR), frozen in isopentane precooled with liquid nitrogen and stored at $-80^{\circ} \mathrm{C}$. Serial sections of $10 \mu \mathrm{m}$-thick were cut in a cryostat, collected on SuperFrost ${ }^{\circledR P l u s}$ microscope slides (Ref. 631-0108, VWR) and stored at $-80^{\circ} \mathrm{C}$. TA and SL sections were processed for hematoxylin and eosin staining using conventional methods to analyse muscle histology. For immunohistochemical identification of muscle fiber types, slides were defrosted and equilibrated at room temperature (RT) for $15 \mathrm{~min}$ and hydrated by washing with PBS 1X. Internal peroxidases were blocked by incubation with $3 \% \mathrm{H}_{2} \mathrm{O}_{2}$ in PBS for 30 min Hydrogen peroxide solution (30\% w/w in $\mathrm{H}_{2} \mathrm{O}$, Ref. $\mathrm{H} 1009$, Sigma-Aldrich) and then washed twice with PBS for 3 min each. M.O.M. Ig blocking reagent (3.6\% in PBS) was used to block unspecific staining for $1 \mathrm{~h}$ at RT (Ref. PK-220, Vector laboratories). Then, they were washed twice with PBS for 3 min and incubated for 1 $h$ at RT with primary antibodies against Type IIA and IIB fibers (Ref. SC-71 and BFF3 respectively, Developmental Studies Hybridoma Bank). Slides were washed for 3 min with $0.1 \%$ Tween PBS and then incubated for 20 min at RT with 1/250 v/v secondary biotinylated $\alpha$-mouse IgG diluted in $8 \% \mathrm{v} / \mathrm{v}$ M.O.M. protein concentrate (Ref. PK-220, Vector laboratories) in PBS 1X. Sections were then washed twice for 3 min with $0.1 \%$ Tween PBS $1 \mathrm{X}$, followed by incubation for 15 min at RT with ABC (avidin/biotin) solution (3.6\% A reagent, 3.6\% B reagent in PBS $1 \mathrm{x}$, mixed and incubated for 30 min prior use) (Ref. PK-220, Vector laboratories). Two washes were performed for 3 min with $0.1 \%$ Tween and finally, the staining was developed by 
incubation with $0.6 \%$ 3,3'-diaminobenzidine in PBS under the microscope until brown coloration was clearly observed. The slides were immediately mounted with DPX (Ref. 44581, Sigma-Aldrich). The secondary antibody specificity was tested by incubation in the absence of primary antibody. Slides were visualized using a Leica DMI 6000 B microscope. Mean cross-sectional area, number of myofibers per area and the percentage of myofibers positive for Type IIA and IIB fibers were determined using ImageJ software $1.48 \mathrm{v}$ version (Wayne Rasband, National Institutes of Health, USA). The percentage of fiber IIX was determined as the difference between the total number of type II fibres and the sum of type IIA and IIB fibers [IIX $=$ II - (IIB + IIA)]. A minimum of 400 myofibers were analyzed for each experiment.

\section{Subcellular fractionation}

Mitochondria, nuclear and cytosolic fractions were isolated from TA muscles as previously described in Dimauro et al., 2012. Briefly $50 \mathrm{mg}$ of tissue was homogenized in STM buffer comprising $250 \mathrm{mM}$ sucrose, $50 \mathrm{mM}$ Tris- $\mathrm{HCl}$ pH 7.4, 5 $\mathrm{mM} \mathrm{MgCl2}$, protease and phosphatase inhibitor cocktails. The homogenate was centrifuged at $800 \mathrm{~g}$ for 15 minutes and the pellet was labelled as $P_{0}$ and kept on ice, the supernatant was labelled as $S_{0}$ and used for subsequent isolation of mitochondrial and cytosolic fractions. The pellet $\mathrm{P}_{0}$ (containing nuclei and debris) was resuspended in STM buffer and centrifuged at $500 \mathrm{~g}$ for 15 minutes. After different purification steps, the pellet was saved as the "nuclear fraction". 
Cytosolic and mitochondrial fractions were extracted from $S_{0}$ by centrifugation at 800 $g$ for 10 minutes. The supernatant $S_{1}$ was saved and the pellet $\left(P_{1}\right)$ was discarded. $\mathrm{S}_{1}$ was centrifuged at $11,000 \mathrm{~g}$ for 10 minutes and the pellet $\mathrm{P}_{2}$ was kept on ice and the supernatant $S_{2}$ (containing cytosol and microsomal fraction) was precipitated in cold $100 \%$ acetone at $-20^{\circ} \mathrm{C}$ for at least 1 hour followed by centrifugation at 12,000 $g$ for 5 minutes. The pellet $\left(P_{3}\right)$ was then resuspended in STM buffer and labelled as "cytosolic fraction". The pellet $\mathrm{P}_{2}$ was resuspended in STM buffer and centrifuged at $11,000 \mathrm{~g}$ for 10 minutes. Once centrifuged, supernatant $\mathrm{S}_{4}$ was discarded, the mitochondrial pellet $\left(\mathrm{P}_{4}\right)$ was resuspended in SOL buffer (comprising: $50 \mathrm{mM}$ Tris $\mathrm{HCl} \mathrm{pH} \mathrm{6.8,} 1 \mathrm{mM}$ EDTA, 0.5\% Triton-X-100, protease and phosphatase inhibitors) by sonication on ice at high setting for $5-10$ seconds with 30 second pauses, 3 times and labelled as "mitochondrial fraction".

The protein content of each compartment was determined using BCA protein assay (Ref. 23225, Pierce ${ }^{\mathrm{TM}}$ BCA Protein Assay Kit). The purity of each fraction was assessed by Western Blot by analyzing the presence of specific protein markers: histone H3 (nuclei), glyceraldehyde 3-phosphate dehydrogenase (GAPDH, cytosol), and cytochrome c oxidase subunit IV (COX IV, mitochondria).

\section{Western blot analysis}

Frozen skeletal muscles were homogenized using a TissueRuptor (Qiagen) in icecold RIPA buffer (150 mM NaCl, 1\% Triton X-100, 0.5\% deoxycholate, $0.1 \%$ SDS and $50 \mathrm{mM}$ Tris $\mathrm{pH} 7.4$ supplemented with $2 \mu \mathrm{g} / \mathrm{ml}$ Aprotinin, $30 \mathrm{mM}$ PMSF, $1 \mathrm{mM}$ Sodium orthovanadate, $5 \mathrm{mM}$ Sodium fluoride (NAF) and $2 \mu \mathrm{g} / \mathrm{ml}$ Pepstatin A). 
Protein lysates were obtained collecting supernatants after centrifugation at $16.000 \mathrm{~g}$ for $30 \mathrm{~min}$ at $4^{\circ} \mathrm{C}$. Protein concentrations were determined by BCA method (Ref. 23225, Pierce ${ }^{\mathrm{TM}}$ BCA Protein Assay Kit). For each sample, 20-40 $\mu \mathrm{g}$ of protein extract were separated by $8-12 \%$ SDS-polyacrylamide electrophoresis and electrotransferred to polyvinylidene difluoride (PVDF) membranes (Ref. IPH00010, Millipore). Total levels of protein transferred to membranes were detected using Sypro Ruby protein blot stain as described by the manufacturer (Ref. S11791, ThermoFisher Scientific). Membranes were blocked in Odyssey blocking buffer for 1 hour (Ref. 927-50000, LI-COR) and probed with the primary antibody overnight at $4^{\circ} \mathrm{C}$. All antibodies were diluted in Odyssey blocking buffer- $0.2 \%$ Tween-20 and information of each antibody used is described in Supplemental Table 1. After incubation, four washes with $0.1 \%$ Tween TBS $1 \mathrm{X}(248 \mathrm{mM}$ Tris, $1.37 \mathrm{M} \mathrm{NaCl}$ and $26.83 \mathrm{mM} \mathrm{KCl}, \mathrm{pH} 8$ ) for $5 \mathrm{~min}$ each were performed. Then, membranes were incubated with the corresponding fluorescently labeled secondary antibodies (Supplemental Table 1) diluted in Odyssey blocking buffer-0.2\% Tween-20 for 60 min at room temperature. After incubation, four washes with $0.1 \%$ Tween TBS $1 \mathrm{X}$ for 5 min each were performed. Membranes were scanned in the appropriate channels (700 nm for IRDye680 antibody, $800 \mathrm{~nm}$ for IRDye800 antibody) in Odyssey CLx Imaging System. The densitometry of the resultant bands was analysed with the Image Studio Lite Ver 5.2 software. $\alpha$-Tubulin was used as loading control, as well as total protein staining (Sypro Ruby protein blot stain, Invitrogen). Specific phosphorylation was determined as the ratio of phosphorylated protein to total protein content. 


\section{Gene expression analysis}

Total RNA from skeletal muscle, heart, brain, lung and spleen tissues was extracted using PureLink ${ }^{\text {TM }}$ RNA Mini Kit (Ref. 12183018A, Ambion, Life Technologies), including ON-Column PureLink®DNase treatment. Following extraction, RNA was quantified using Nanodrop (ThermoScientific) and integrity was checked by observation of $28 \mathrm{~S}$ and $18 \mathrm{~S}$ ribosomic bands on $1 \%$ agarose gel electrophoresis stained with ethidium bromide. For each sample, 500ng of extracted RNA was then reverse transcribed by SuperScript TM III. RNA expression levels were analyzed using the quantitative Real Time PCR technique in the LightCycler®480 (Roche Diagnostics Corporation) platform. Each sample was run in triplicate. Samples without reverse transcriptase during cDNA conversion were also assessed to ensure that there was no DNA contamination. Two or three different references genes were used for each experiment (Tbp1, Actb, Sdha).

Primers were designed using the Integrated DNA Technologies (IDT) tool (http://eu.idtdna.com/scitools/Applications/RealTimePCR/) and are listed in Supplemental Table 2. Primer efficiency was calculated by extracting fluorescence raw data and using the Chainy tool (http://maplab.imppc.org/chainy/; [51]).

\section{Mitochondrial DNA content}

For total DNA extraction, frozen skeletal muscles were homogenized using a TissueRuptor (Qiagen) in the following digestion buffer: $50 \mathrm{mM}$ Tris- $\mathrm{HCl} \mathrm{pH} 8 ; 5 \mathrm{mM}$ EDTA; $1 \%$ SDS ans $200 \mathrm{nM} \mathrm{NaCl}$ and $1 \mathrm{mg} / \mathrm{ml}$ Proteinase $\mathrm{K}$. Then, samples were incubated at $55^{\circ} \mathrm{C}$ overnight with shaking. DNA was purified by phenol/chloroform 
extraction and precipitated afterwards in absolute ethanol in presence of $0.4 \mathrm{M}$ of sodium acetate. The pellet was washed twice in ethanol $70 \%$ and finally resuspended in TE buffer $(10 \mathrm{mM}$ Tris- $\mathrm{HCl} \mathrm{pH} 8$ and $1 \mathrm{mM}$ EDTA. DNA purity was checked and quantified by NanoDrop and 100ng of DNA was run on 1\% agarose gel stained with ethidium bromide to check DNA integrity. RT-qPCR analyses were performed to measure the mitochondrial DNA (mtDNA) content in WT and HDAC11 ${ }^{-}$ 1- mice. mtDNA content was expressed as the ratio of the CT value from the mitochondrial-encoded Cox2 gene and a mitochondrial DNA fragment $(f-m t D N A)$ to Ct values from the genomic-encoded Sdha and Rplpo genes. The primers are detailed in Supplemental Table 3.

\section{Determination of acylcarnitine content}

Frozen skeletal muscles were homogenized using a TissueRuptor (Qiagen) in 0.2M $\mathrm{NaCl}$ buffer and protein concentrations were determined by BCA method (Ref. 23225, Pierce ${ }^{\mathrm{TM}}$ BCA Protein Assay Kit). Acylcarnitines were analyzed using an Acquity UPLC-TOF system (Waters) with a BEH C8 column (1.7 $\mu \mathrm{m}$ particle size, $100 \mathrm{~mm} \times 2.1 \mathrm{~mm}$, Waters). The two mobile phases were $1 \mathrm{mM}$ ammonium formate in methanol (phase A) and $2 \mathrm{mM}$ ammonium formate in $\mathrm{H} 2 \mathrm{O}$ (phase $\mathrm{B}$ ), both phases with $0.05 \mathrm{mM}$ formic acid. The following gradient was programmed: $0 \mathrm{~min}, 65 \% \mathrm{~A} ; 10 \mathrm{~min}, 90 \% \mathrm{~A} ; 15 \mathrm{~min}, 99 \% \mathrm{~A} ; 17 \mathrm{~min}, 99 \% \mathrm{~A} ; 20 \mathrm{~min}, 65 \% \mathrm{~A}$, and $\mathrm{a}$ flow rate of $0.3 \mathrm{~mL} \min -1$. Quantification was carried out using the extracted ion chromatogram of each compound, with 50-mDa windows. The linear dynamic range 
was determined by injecting standard mixtures. Positive identification of compounds was based on the accurate mass measurement with an error $<5 \mathrm{ppm}$ and their LC retention time was compared to that of a standard $( \pm 2 \%)$.

\section{Fatty acid oxidation assay}

Fatty acid oxidation assay kit (Cat \#: E-141, Biomedical research service center, University at Buffalo, State University of New York) was used to assess the fatty acid $\beta$-oxidation activity levels based on the oxidation of octanoyl-CoA, which is coupled to NADH-dependent reduction of INT to INT-formazan. Frozen skeletal muscles were homogenized using a TissueRuptor (Qiagen) in ice-cold 1X Cell lysis solution provided by the kit. Then, protein concentrations were determined by BCA method (Ref. 23225, Pierce ${ }^{\mathrm{TM}}$ BCA Protein Assay Kit). Sample protein concentration was normalized by diluting with ice-cold $1 \mathrm{X}$ Cell lysis solution to $1.5 \mu \mathrm{g} / \mu \mathrm{l} .10 \mu \mathrm{l}$ of each protein sample was added to a 96-well plate in duplicate and then, $50 \mu \mathrm{l}$ of the control solution $\left(\mathrm{H}_{2} \mathrm{O}\right.$ and $\mathrm{FAO}$ assay solution) was added to one set of wells and 50 $\mu \mathrm{l}$ of the reaction solution (substrate and FAO assay solution) to another set of wells. After mixing contents by gentle agitation for $30 \mathrm{sec}$ the plate was incubate in a humidified $37^{\circ} \mathrm{C}$ incubator for $1 \mathrm{~h}$. The assay was stopped by adding $50 \mu \mathrm{l}$ of $3 \%$ acetic acid to each well followed by brief gentle agitation. FAO activity was

measured at O.D.492 $\mathrm{nm}$ in a SpectroStar NANO query (BMG Labtech). Final values were obtained by subtracting control well reading from reaction well reading for each sample. The subtracted O.D. reading was proportional to fatty acid oxidation activity of the sample. 


\section{Functional assessments}

Non-invasive muscle function tests were used to evaluate muscle function: treadmill exhaustion, grip strength and the four limbs hanging test (Kondziella's inverted screen test).

Muscle endurance was determined by allowing mice run on a treadmill until exhaustion (Panlab LE 8205). Mice were acclimated with a fixed $20 \%$ slope at a speed of $12 \mathrm{~m} / \mathrm{min}$ for $10 \mathrm{~min}$ in 3 constitutive days before the test day. On the exercise-testing day, mice were run at $12 \mathrm{~m} / \mathrm{min}$ for $10 \mathrm{~min}$, and speed was increased by $2 \mathrm{~m} / \mathrm{min}$ every 2 min until mice were exhausted. Running time, speed, and distance were recorded when the mice failed to climb the treadmill in $3 \mathrm{~s}$ despite the electric shock. Work and power were calculated as the following formula: work $(\mathrm{SI})=$ bodyweight $(\mathrm{kg}) \times$ gravity $(9.81 \mathrm{~m} / \mathrm{s} 2) \times$ vertical speed $(\mathrm{m} / \mathrm{s} \times$ angle $) \times$ time $(\mathrm{s})$,

power $(\mathrm{W})=$ work $(\mathrm{SI}) /$ time $(\mathrm{s})$ [52-55]. Grip strength was used to test maximal isometric grip strength in WT and $\mathrm{HDAC} 11^{--}$mice. Mice were held by the tail and allowed them to place in the wire screen connected to the force sensor, and then slowly pulled away until they released. Each test was performed three times, with a $60 \mathrm{~s}$ rest between trials; the mean of these three trials was used for the analysis [56]. The four limb-hanging test measure the ability of the mice to hang upside down from a wire screen and was tested as previously described $[57,58]$. Briefly, mouse was placed in the centre of the wire mesh screen and the screen was rotated to an inverted position over $2 \mathrm{sec}$, with the mouse's head declining first. It was recorded the time that the mouse was capable of hanging. Each mouse was given three trials, with a 2 min rest between trials, unless the mice was able to hanging during $=$ or $>$ 
than 10 minutes (in this case it was only recorded 1 trial). The maximum hanging time (i.e. the longest of the trials) was recorded and used for the analysis. The experiments were performed during 5 non-consecutive days and the same operator performed the test for all the animals involved in the study.

\section{Statistical analysis}

Statistical significance between WT and HDAC11 $11^{-1}$ was determined by two-tailed unpaired Student's t test. Statistical significance of HDAC11 expression between different tissues and muscles was calculated by ANOVA. The statistical package included in GraphPad Prism 6 software was used to assess the statistical significance. The data in the graphs represent the mean \pm SD of independent experiments. A p value $<0.05$ was considered significant $\left({ }^{*} p<0.05\right.$; ${ }^{* *} p<$ $\left.0.001 ;{ }^{* * *} \mathrm{p}<0.0001\right)$. 


\section{Acknowledgments}

We thank Rosa Mํㅡㄹ Ampudia i Dr. Sara Capdevila for excellent technical assistance. We thank Drs. Dolors Serra, Pura Muñoz-Cánoves and Coral Sanfeliu for kindly providing ACC/pACC and TOM20, AMPK/pAMPK and COXIV antibodies, respectively. We thank Drs. Pura Muñoz-Cánoves and Monserrat Batlle for sharing with us the grip strength and treadmill equipments, respectively. Finally, we are also very grateful to Drs. Dolors Serra and Pura Muñoz-Cánoves for their advices and helpful discussions. This work was supported by Ministerio de Economía y Competitividad (BFU2016-80748 to M.S. and BFU2017-89408-R to A.M.P.) and Ministerio de Ciencia, Innovación y Universidades (RTI2018-094009-B-I00 to M.A.P.), Feder funds, Generalitat de Catalunya (2017 SGR969 and 2017 SGR206) and Junta de Castilla y Leon (CSI239P18). E.H. was supported in part by BFU201680748 and Y.N.A was supported by FPU12/05668.

\section{Author contributions}

E. Hurtado and Y. Núñez-Álvarez designed and carried out experiments and data analysis. M Muñoz gave technical assistance. C. Gutiérrez-Caballero and A. M. Pendás generated the HDAC11-deficient mice. J. Casas performed the acylcarnitines assays. M.A. Peinado revised the manuscript. M. Suelves conceived the experiments and wrote the manuscript. All the authors contributed with critical discussions and read and approved the final version of the manuscript. 


\section{Disclosure of potential conflicts of interest}

The authors declare that they have not competing interest.

\section{References}

1. Schiaffino S, Reggiani C (2011) Fiber Types in Mammalian Skeletal Muscles. Physiol Rev 91: 1447-1531.

2. Bassel-Duby R, Olson EN (2006) Signaling Pathways in Skeletal Muscle Remodeling. Annu Rev Biochem 75: 19-37.

3. Gao L, Cueto MA, Asselbergs F, Atadja P (2002) Cloning and functional characterization of HDAC11, a novel member of the human histone deacetylase family. J Biol Chem 277: 25748-25755.

4. Haberland M, Montgomery RL, Olson EN (2009) The many roles of histone deacetylases in development and physiology: implications for disease and therapy. Nat Rev Genet 10: 32-42.

5. Hull EE, Montgomery MR, Leyva KJ (2016) HDAC Inhibitors as Epigenetic Regulators of the Immune System: Impacts on Cancer Therapy and Inflammatory Diseases. Biomed Res Int 2016: 8797206.

6. Shakespear MR, lyer A, Cheng CY, Das Gupta K, Singhal A, Fairlie DP, Sweet MJ (2018) Lysine Deacetylases and Regulated Glycolysis in Macrophages. Trends Immunol 39: 473-488.

7. Kutil Z, Novakova Z, Meleshin M, Mikesova J, Schutkowski M, Barinka C (2018) Histone Deacetylase 11 Is a Fatty-Acid Deacylase. ACS Chem Biol 13: 685-693.

8. Moreno-Yruela C, Galleano I, Madsen AS, Olsen CA (2018) Histone Deacetylase 11 Is an $\varepsilon$-N-Myristoyllysine Hydrolase. Cell Chem Biol 25: 849856.e8.

9. Cao J, Sun L, Aramsangtienchai P, Spiegelman NA, Zhang X, Huang W, Seto $\mathrm{E}$, Lin H (2019) HDAC11 regulates type I interferon signaling through defattyacylation of SHMT2. Proc Natl Acad Sci 116: 5487-5492. 
10. Sun L, Marin de Evsikova C, Bian K, Achille A, Telles E, Pei H, Seto E (2018) Programming and Regulation of Metabolic Homeostasis by HDAC11. EBioMedicine 33: 157-168.

11. Bagchi RA, Ferguson BS, Stratton MS, Hu T, Cavasin MA, Sun L, Lin Y-H, Liu D, Londono P, Song K, et al. (2018) HDAC11 suppresses the thermogenic program of adipose tissue via BRD2. JCl Insight 3:.

12. Wu H, Naya FJ, McKinsey TA, Mercer B, Shelton JM, Chin ER, Simard AR, Michel RN, Bassel-Duby R, Olson EN, et al. (2000) MEF2 responds to multiple calcium-regulated signals in the control of skeletal muscle fiber type. EMBO $J$ 19: 1963-1973.

13. Naya FJ, Mercer B, Shelton J, Richardson JA, Williams RS, Olson EN (2000) Stimulation of Slow Skeletal Muscle Fiber Gene Expression by Calcineurin in Vivo. J Biol Chem 275: 4545-4548.

14. McCullagh KJA, Calabria E, Pallafacchina G, Ciciliot S, Serrano AL, Argentini C, Kalhovde JM, Lomo T, Schiaffino S (2004) NFAT is a nerve activity sensor in skeletal muscle and controls activity-dependent myosin switching. Proc Natl Acad Sci 101: 10590-10595.

15. Kivelä R, Salmela I, Nguyen YH, Petrova T V., Koistinen HA, Wiener Z, Alitalo $\mathrm{K}$ (2016) The transcription factor Prox1 is essential for satellite cell differentiation and muscle fibre-type regulation. Nat Commun 7: 13124.

16. Chan MC, Arany Z (2014) The many roles of PGC-1a in muscle--recent developments. Metabolism 63: 441-451.

17. Joshi P, Greco TM, Guise AJ, Luo Y, Yu F, Nesvizhskii Al, Cristea IM (2013) The functional interactome landscape of the human histone deacetylase family. Mol Syst Biol 9: 1-21.

18. Keedy KS, Archin NM, Gates AT, Espeseth A, Hazuda DJ, Margolis DM (2009) A Limited Group of Class I Histone Deacetylases Acts To Repress Human Immunodeficiency Virus Type 1 Expression. J Virol 83: 4749-4756.

19. Bonnefont J, Djouadi F, Prip-Buus C, Gobin S, Munnich A, Bastin J (2004) Carnitine palmitoyltransferases 1 and 2: biochemical, molecular and medical aspects. Mol Aspects Med 25: 495-520. 
20. Jogl G, Hsiao Y-S, Tong L (2004) Structure and Function of Carnitine Acyltransferases. Ann N Y Acad Sci 1033: 17-29.

21. Chen Z-P, McConell GK, Michell BJ, Snow RJ, Canny BJ, Kemp BE (2000) AMPK signaling in contracting human skeletal muscle: acetyl-CoA carboxylase and NO synthase phosphorylation. Am J Physiol Metab 279: E1202-E1206.

22. Zurlo F, Lillioja S, Esposito-Del Puente A, Nyomba BL, Raz I, Saad MF, Swinburn BA, Knowler WC, Bogardus C, Ravussin E (1990) Low ratio of fat to carbohydrate oxidation as predictor of weight gain: study of 24-h RQ. Am J Physiol Metab 259: E650-E657.

23. Gregoretti I, Lee Y-M, Goodson H V (2004) Molecular Evolution of the Histone Deacetylase Family: Functional Implications of Phylogenetic Analysis. $\mathrm{J} \mathrm{Mol}$ Biol 338: 17-31.

24. Rakus D, Gizak A, Deshmukh A, Wïniewski JR (2015) Absolute quantitative profiling of the key metabolic pathways in slow and fast skeletal muscle. $J$ Proteome Res 14: 1400-1411.

25. Potthoff MJ, Wu H, Arnold MA, Shelton JM, Backs J, McAnally J, Richardson JA, Bassel-Duby R, Olson EN (2007) Histone deacetylase degradation andMEF2 activation promote the formation of slow-twitch myofibers. J Clin Invest 117: 2459.

26. Cohen TJ, Choi M-C, Kapur M, Lira VA, Yan Z, Yao T-P (2015) HDAC4 regulates muscle fiber type-specific gene expression programs. Mol Cells $\mathbf{3 8}$ : 343-348.

27. Miska EA, Karlsson C, Langley E, Nielsen SJ, Pines J, Kouzarides T (1999) HDAC4 deacetylase associates with and represses the MEF2 transcription factor. EMBO J 18: 5099-5107.

28. McKinsey TA, Zhang C-L, Lu J, Olson EN (2000) Signal-dependent nuclear export of a histone deacetylase regulates muscle differentiation. Nature 408 : 106-111.

29. McKinsey TA, Zhang CL, Olson EN (2002) MEF2: a calcium-dependent regulator of cell division, differentiation and death. Trends Biochem Sci 27: $40-47$. 
30. Chang S, McKinsey TA, Zhang CL, Richardson JA, Hill JA, Olson EN (2004) Histone deacetylases 5 and 9 govern responsiveness of the heart to a subset of stress signals and play redundant roles in heart development. Mol Cell Biol 24: 8467-8476.

31. Black BL, Olson EN (1998) Transcriptional control of muscle development by myocyte enhancer factor-2 (MEF2) proteins. Annu Rev Cell Dev Biol 14: 167196.

32. Seto E, Yoshida M (2014) Erasers of histone acetylation: the histone deacetylase enzymes. Cold Spring Harb Perspect Biol 6: a018713.

33. Tong JK, Hassig CA, Schnitzler GR, Kingston RE, Schreiber SL (1998) Chromatin deacetylation by an ATP-dependent nucleosome remodelling complex. Nature 395: 917-921.

34. Xue Y, Wong J, Moreno GT, Young MK, Côté J, Wang W (1998) NURD, a novel complex with both ATP-dependent chromatin-remodeling and histone deacetylase activities. Mol Cell 2: 851-861.

35. You A, Tong JK, Grozinger CM, Schreiber SL (2001) CoREST is an integral component of the CoREST- human histone deacetylase complex. Proc Natl Acad Sci 98: 1454-1458.

36. Bantscheff M, Hopf C, Savitski MM, Dittmann A, Grandi P, Michon A-M, Schlegl J, Abraham Y, Becher I, Bergamini G, et al. (2011) Chemoproteomics profiling of HDAC inhibitors reveals selective targeting of HDAC complexes. Nat Biotechnol 29: 255-265.

37. Cheng F, Lienlaf M, Perez-Villarroel P, Wang HW, Lee C, Woan K, Woods D, Knox T, Bergman J, Pinilla-lbarz J, et al. (2014) Divergent roles of histone deacetylase 6 (HDAC6) and histone deacetylase 11 (HDAC11) on the transcriptional regulation of IL10 in antigen presenting cells. Mol Immuno/ 60: 44-53.

38. Hubbert $C$, Guardiola A, Shao R, Kawaguchi $Y$, Ito A, Nixon A, Yoshida M, Wang X-F, Yao T-P (2002) HDAC6 is a microtubule-associated deacetylase. Nature 417: 455-458.

39. Valenzuela-Fernández A, Cabrero JR, Serrador JM, Sánchez-Madrid F (2008) 
HDAC6: a key regulator of cytoskeleton, cell migration and cell-cell interactions. Trends Cell Biol 18: 291-297.

40. Lin J, Wu H, Tarr PT, Zhang C-Y, Wu Z, Boss O, Michael LF, Puigserver P, Isotani E, Olson EN, et al. (2002) Transcriptional co-activator PGC-1a drives the formation of slow-twitch muscle fibres. Nature 418: 797-801.

41. Arany Z, He H, Lin J, Hoyer K, Handschin C, Toka O, Ahmad F, Matsui T, Chin S, Wu P-H, et al. (2005) Transcriptional coactivator PGC-1a controls the energy state and contractile function of cardiac muscle. Cell Metab 1: 259271.

42. Ringel AE, Tucker SA, Haigis MC (2018) Chemical and Physiological Features of Mitochondrial Acylation. Mol Cell 72: 610-624.

43. Jiang H, Khan S, Wang Y, Charron G, He B, Sebastian C, Du J, Kim R, Ge E, Mostoslavsky R, et al. (2013) SIRT6 regulates TNF- $\alpha$ secretion through hydrolysis of long-chain fatty acyl lysine. Nature 496: 110-113.

44. Jing $H$, Zhang $X$, Wisner SA, Chen $X$, Spiegelman NA, Linder ME, Lin $H$ (2017) SIRT2 and lysine fatty acylation regulate the transforming activity of KRas4a. Elife 6:.

45. Anderson DD, Quintero CM, Stover PJ (2011) Identification of a de novo thymidylate biosynthesis pathway in mammalian mitochondria. Proc Natl Acad Sci 108: 15163-15168.

46. Rinaldo P, Matern D, Bennett MJ (2002) Fatty Acid Oxidation Disorders. Annu Rev Physio/ 64: 477-502.

47. Gumucio JP, Qasawa AH, Ferrara PJ, Malik AN, Funai K, McDonagh B, Mendias CL (2019) Reduced mitochondrial lipid oxidation leads to fat accumulation in myosteatosis. FASEB J 33: 7863-7881.

48. Martin MW, Lee JY, Lancia DR, Ng PY, Han B, Thomason JR, Lynes MS, Marshall CG, Conti C, Collis A, et al. (2018) Discovery of novel N-hydroxy-2arylisoindoline-4-carboxamides as potent and selective inhibitors of HDAC11. Bioorganic Med Chem Lett 28: 2143-2147.

49. Son SI, Cao J, Zhu C-L, Miller SP, Lin H (2019) Activity-Guided Design of HDAC11-Specific Inhibitors. ACS Chem Biol 14: 1393-1397. 
50. Gutiérrez C (2012) Análisis funcional de proteínas implicadas en el mantenimiento de la estabilidad cromosómuca en mamíferos.

51. Mallona I, Díez-Villanueva A, Martín B, Peinado MA (2017) Chainy, an universal tool for standardized relative quantification in real-time PCR. Bioinformatics 33: btw839.

52. Bi P, Yue F, Sato Y, Wirbisky S, Liu W, Shan T, Wen Y, Zhou D, Freeman J, Kuang $S$ (2016) Stage-specific effects of Notch activation during skeletal myogenesis. Elife 5:.

53. Nie Y, Sato Y, Wang C, Yue F, Kuang S, Gavin TP (2016) Impaired exercise tolerance, mitochondrial biogenesis, and muscle fiber maintenance in miR133a-deficient mice. FASEB J 30: 3745-3758.

54. Yue F, Bi P, Wang C, Li J, Liu X, Kuang S (2016) Conditional Loss of Pten in Myogenic Progenitors Leads to Postnatal Skeletal Muscle Hypertrophy but Age-Dependent Exhaustion of Satellite Cells. Cell Rep 17: 2340-2353.

55. Sato $\mathrm{Y}$, Ohtsubo $\mathrm{H}$, Nihei $\mathrm{N}$, Kaneko $\mathrm{T}$, Sato $\mathrm{Y}$, Adachi S-I, Kondo S, Nakamura M, Mizunoya W, lida $\mathrm{H}$, et al. (2018) Apobec2 deficiency causes mitochondrial defects and mitophagy in skeletal muscle. FASEB J 32: 1428.

56. Bonetto A, Andersson DC, Waning DL (2015) Assessment of muscle mass and strength in mice. Bonekey Rep 4: 1-10.

57. Kondziella W (1964) A new method for the measurement of muscle relaxation in white mice. Arch Int Pharmacodyn Ther 152: 277-284.

58. McDonald MP, Miller KM, Li C, Deng C, Crawley JN (2001) Motor deficits in fibroblast growth factor receptor-3 null mutant mice. Behav Pharmacol 12: 477-486. 


\section{Figure Legends}

Figure 1. HDAC11 deficiency does not affect muscle structure and growth.

A Quantification of Hdac11 mRNA levels by RT-qPCR in skeletal muscle (Sk muscle), heart, brain, lung and spleen.

B Confirmation by PCR of WT $(+/+)$, heterozygote (+/-) and HDAC11 KO (-/-) mice genotypes. Image comparing $\mathrm{WT}$ and $\mathrm{HDAC} 11^{-/}$adult mice and representation of the body weight.

C Image shows Soleus (SL), Extensor Digitorum Longus (EDL), Plantaris (PL), Tibialis Anterior (TA) and Gastrocnemius (GC) muscles and their weight is represented.

D Left: Hematoxylin/eosin (H/E) representative sections of TB and SL muscles from WT and HDAC11/- male mice. Right: Average values of male and female myofiber cross-sectional area (CSA) and number of myofiber $/ \mathrm{mm}^{2}$. Scale bar: $100 \mu \mathrm{m}$.

Data information: $n=4-6$ mice for each group. In (D) a minimum of 400 myofibers were analyzed per muscle and animal. Data are represented as means \pm SD. Statistical significance was determined in (A) by ANOVA (Sk muscle as a reference value) and in (B-D) by Student's t test ( ${ }^{*} p$ value $\left.<0.05,{ }^{* *} p<0.01 ;{ }^{* * *} p<0.001\right)$.

\section{Figure 2. HDAC11 deficiency promotes a glycolytic-to-oxidative myofiber transition.}

A Quantification of Hdac11 mRNA levels by RT-qPCR in TA and EDL muscles (fast-twitch) compared with SL muscle (slow-twitch).

$B$ WB analysis and quantitative results of HDAC11 in TA and SL muscles from WT mice.

C WB analysis and quantitative results of MyHC-IIB and MyHC-IIX/IIA in TA muscle from WT and HDAC $11^{-/}$mice.

D Representative sections and quantification of $\mathrm{MyHC}$-IIB and MyHC-IIA immunostainning in TA muscle from WT and $\mathrm{HDAC} 11^{-/}$mice. The percentage 
of fiber IIX was determined as the difference between the total number of type II fibres and the sum of type IIA and IIB fibers [IIX = II - (IIB + IIA)]. Scale bar: $75 \mu \mathrm{m}$.

Data information: $n=4-6$ mice for each group. In (D) a minimum of 400 myofibers were analyzed per muscle and animal. Data are represented as means \pm SD. Statistical significance was determined in (A) by ANOVA and in (B-D) by Student's $t$ test $\left({ }^{*} p\right.$ value $\left.<0.05,{ }^{* *} p<0.01 ;{ }^{* * *} p<0.001\right)$.

Figure 3. Mitochondrial content is increased in $\mathrm{HDAC}_{11^{-/}}$skeletal muscle.

A Quantification of Myoglobin (Mb) mRNA levels by RT-qPCR in WT and HDAC $11^{-1 /}$ skeletal muscles.

B WB showing the protein levels of mitochondrial coactivator PGC-1 $\alpha$ in WT and HDAC $11^{-/}$skeletal muscles.

C Quantification of mitochondrial DNA (mtDNA) by RT-qPCR (Cox2 and a DNA fragment named $f-m t D N A$ ) normalized to genomic DNA (Sdha gene).

D WB analysis and quantification of the mitochondrial markers TOM20, COXIV and mtTFA.

E WB analysis of the subcellular fractionation performed in skeletal muscle of WT mice. COXIV, GAPDH and H3 markers were used to validate fraction purity (mitochondria, cytosol and nucleus, respectively).

F Colocalization of HDAC11 (in green) and mtTFA (in red) in TA muscles from WT mice by confocal microscopy. All nuclei are counterstained by DAPI (blue). The dotted white line indicates the inset of the corresponding picture. Arrows indicate colocalization between HDAC11 and mtTFA in mitochondria, as indicated by the yellow color. Scale bar: $20 \mu \mathrm{m}$, scale bar Inset: $10 \mu \mathrm{m}$.

Data information: $n=4-6$ mice for each group. Data are represented as means \pm SD. Statistical significance was determined by Student's t test ( ${ }^{*} p$ value $<0.05$ ).

Figure 4. HDAC11 deficiency enhances fatty acid oxidation through the activation of AMPK-ACC signaling pathway. 
A WB showing the protein levels of AMPK ${ }^{\text {Thr172, }}$, total AMPK, ACC ${ }^{\text {Ser79 }}$ and total ACC in WT and HDAC11\% skeletal muscles. Quantification is represented as the ratio of phosphorylated to total protein.

B Acylcarnitine levels were measured by HPLC-MS/M in WT and HDAC11-skeletal muscles. Left: total acylcarnitine levels. Right: acylcarnitine levels classified by species.

C Measurement of fatty acid $\beta$-oxidation activity in WT and HDAC $11^{-/}$skeletal muscles.

Data information: $n=4-6$ mice for each group. Data are represented as means \pm SD. Statistical significance was determined by Student's $t$ test $\left({ }^{*} p\right.$ value $<0.05$, ${ }^{* *} p<$ $\left.0.01 ;{ }^{* * *} \mathrm{p}<0.001\right)$.

Figure 5. HDAC11-deficient mice show better muscle performance.

A Treadmill exhaustion test was performed with progressive speed and time, with a $20 \%$ slope and speed and distance were quantified until exhaustion. Work and power were calculated with the normalization of individual body weight.

B Grip Strength test in WT and HDAC $11^{-/}$mice.

C Kondziella's inverted screen test was performed in WT and $\mathrm{HDAC} 11^{-/}$mice during 5 non consecutive days. Left: average of the maximum hanging time of all 5 days. Right: maximum hanging time per day.

Data information: $\mathrm{n}=6-8$ mice for each group. Data are represented as means \pm SD. Statistical significance was determined by Student's $t$ test $\left({ }^{*} p\right.$ value $<0.05,{ }^{* *} p<$ $0.01)$.

Figure 6. Schematic representation of HDAC11 functions in skeletal muscle tissue.

HDAC11 is required for balancing fiber type composition (1) and for modulating 
mitochondrial lipid oxidation (2) in skeletal muscle. Lack of HDAC11 results in increased number of oxidative myofibers accompanied by an increase of myoglobin levels and mitochondrial mass, suggesting an enhanced oxidative capacity in skeletal muscle. In addition, HDAC11 depletion increases mitochondrial fatty acid $\beta$ oxidation through the activation of the AMPK-ACC signaling pathway and consequently, acylcarnitine levels are reduced in muscle tissue. The functional consequence of HDAC11 deficiency is the improvement of muscle strength and fatigue resistance, indicating a better skeletal muscle performance.

\section{Supplemental Figure 1. Generation and validation of $\mathrm{HDAC}_{11^{-/-}}$mice.}
A Schematic representation of the strategy used for generating $\mathrm{HDAC} 11^{-/}$mice.
B WB analysis of HDAC11 expression from WT and HDAC11 $1{ }^{-1 /}$ brain samples. Total protein staining was used as a loading control.
C Observed and expected Mendelian ratio from heterozygote HDAC11 crosses.

\section{Supplemental Figure 2. HDAC11-deficiency does not alter the expression levels of slow- and fast-related muscle genes.}

Quantification of mRNA levels by RT-qPCR of different essential genes for slow and fast fiber-type determination such us transcription factors, sarcomeric proteins and calcium regulators in TA muscle. $\mathrm{n}=5$ mice for each group. Data are represented as means $\pm S D$ and $W T$ expression reference value $=1$. Statistical significance was determined by Student's $t$ test.

\section{Supplemental Figure 3. Mitochondrial content is increased in HDAC11 ${ }^{-/-}$ skeletal muscle.}

A Quantification of mitochondrial DNA (mtDNA) by RT-qPCR (Cox2 and a DNA fragment named f-mtDNA) normalized to genomic DNA (Rp/p0 gene). 
B Quantification of mtTbf2 mRNA by RT-qPCR in WT and HDAC11 $1^{-}$skeletal muscles.

Data information: $n=4-6$ mice for each group. Data are represented as means \pm SD. Statistical significance was determined by Student's $t$ test ( ${ }^{*} p$ value $<0.05$ ). 
Supplemental Table 1. List of antibodies used for Western blot detection.

\begin{tabular}{|c|c|c|c|c|c|}
\hline Antigen & Conjugate & Host & Dilution & Source & Reference \\
\hline HDAC11 & & Rabbit & $1 / 500$ & Sigma & H4539 \\
\hline HDAC11 & & Mouse & $1 / 400$ & Santa Cruz & sc390737 \\
\hline a-Tubulin & & Mouse & $1 / 10000$ & Sigma & T6074 \\
\hline Fiber type IIB & & Mouse & $1 / 100$ & $\mathrm{DSHB}^{*}$ & BF-F3 \\
\hline $\begin{array}{c}\text { Fiber type } \\
\text { IIA/IIX }\end{array}$ & & Mouse & $1 / 100$ & DSHB* $^{*}$ & A4.74 \\
\hline$P G C 1 \alpha$ & & Mouse & $1 / 1000$ & ProteinTech & 66369 \\
\hline TOM20 & & Mouse & $1 / 500$ & Santa Cruz & sc17764 \\
\hline CoxIV & & Rabbit & $1 / 1000$ & Abcam & 33985 \\
\hline mtTFA & & Mouse & $1 / 400$ & Santa Cruz & sc376672 \\
\hline GAPDH & & Mouse & $1 / 6000$ & ProteinTech & 60004 \\
\hline H3 & & Rabbit & $1 / 10000$ & Abcam & 1791 \\
\hline AMPK & & Rabbit & $1 / 1000$ & Cell Signalling & 2532 \\
\hline AMPK $^{\text {Thr172 }}$ & & Rabbit & $1 / 1000$ & Cell Signalling & 2531 \\
\hline ACC & & Rabbit & $1 / 1000$ & Cell Signalling & 3662 \\
\hline$A C C^{\text {Ser79 }}$ & & Rabbit & $1 / 1000$ & Cell Signalling & 3661 \\
\hline Mouse IgG & IRDye $800 \mathrm{CW}$ & Goat & $1 / 10000$ & LICOR & $926-32210$ \\
\hline Mouse IgG & IRDye 680RD & Goat & $1 / 10000$ & LICOR & $926-68070$ \\
\hline Rabbit IgG & IRDye $800 \mathrm{CW}$ & Goat & $1 / 10000$ & LICOR & $926-32211$ \\
\hline Rabbit IgG & IRDye 680RD & Goat & $1 / 10000$ & LICOR & $926-68071$ \\
\hline
\end{tabular}




\section{Supplemental Table 2. List of primers sequences used for mouse RT-qPCR analysis of mRNA expression.}

\begin{tabular}{|c|c|c|c|}
\hline $\begin{array}{l}\text { Gene } \\
\text { name }\end{array}$ & $F\left(5^{\prime}->3^{\prime}\right)$ & $R\left(5^{\prime}->3^{\prime}\right)$ & $\begin{array}{l}\text { Amplicon } \\
\text { lenght (bp) }\end{array}$ \\
\hline Hdac11 & TTACAACCGCCACATCTACC & GACATTCCTCTCCAССТTCTC & 118 \\
\hline Mef2c & CCAGATCTCCGCGTTCTTATC & CCTCCCATTCCTTGTCCTG & 149 \\
\hline Mef2d & TСТАСССАСТСGСТCACC & GAACTGAAGGCTGGTAAGGAG & 149 \\
\hline Nfatc1 & TGGGAGATGGAAGCAAAGAC & ATAGAAACTGACTTGGACGGG & 114 \\
\hline Prox 1 & AATGCAGGAAGGGCTATCAC & CACGGAAATTGCTGAACCAC & 161 \\
\hline Tnni1 & ATGCCGGAAGTTGAGAGGAAA & TCCGAGAGGTAACGCACCTT & 140 \\
\hline Tnnc1 & GCGGTAGAACAGTTGACAGAG & CCAGCTCCTTGGTGCTGAT & 103 \\
\hline Serca2 & GAGAACGCTCACACAAAGACC & CAATTCGTTGGAGCCCCAT & 120 \\
\hline Six 1 & CCСАСТСАССТCCAGTTTG & GCAAAGAACCCCAGAAACAAG & 157 \\
\hline Tnni2 & ААCTACCTGTCAGAACACTGC & AGCTTCTGGTTCATGTCTTCC & 173 \\
\hline Tnnc2 & AGATGAAAGAGGATGCGAAGG & AGAAGCCCGGAAAATCTCAG & 122 \\
\hline Serca1 & TTACCCCAGACCAAGTTAAGC & TTCGCCTTCCTCAAACCAG & 176 \\
\hline $\operatorname{Sin}$ & ATCACCGTTCTCCTTATGTGG & CATTGTGAGTGTGGGTCAGAG & 162 \\
\hline$M b$ & ССTCTAGCCCAATCACACG & CATCTGCTCCAAAGTCCCC & 118 \\
\hline mtTfb2 & GATAGCGACTCCTAAAAGACCG & ACTGATTCCCCGTGCTTTG & 156 \\
\hline Tbp1 & GGGAGAATCATGGACCAGAA & CCGTAAGGCATCATTGGACT & 113 \\
\hline Actb & GATTACTGCTCTGGCTCCTAG & GACTCATCGTACTCCTGCTTG & 147 \\
\hline Sdha & AGTGCGGGTCGATGAGTATGAT & TATGAGGGGAAACGCAGGTAAG & 176 \\
\hline
\end{tabular}


Supplemental Table 3. List of primers sequences used for mouse RT-qPCR analysis of gene expression.

\begin{tabular}{cccc}
$\begin{array}{c}\text { Gene } \\
\text { name }\end{array}$ & $\mathbf{F}\left(\mathbf{5}^{\prime}-\mathbf{>} \mathbf{3}^{\prime}\right)$ & $\mathbf{R}\left(\mathbf{5}^{\prime}-\mathbf{3} \mathbf{3}^{\prime}\right)$ & $\begin{array}{c}\text { Amplicon } \\
\text { lenght (bp) }\end{array}$ \\
\hline Cox2 & CTACAAGACGCCACAT & GAGAGGGGAGAGCAAT & 217 \\
$f$-mtDNA & ACCGCAAGGGAAAGATGAAA & AGGTAGCTCGTTTGGTTTCGG & 140 \\
Sdha & TACTACAGCCCCAAGTCT & TGGACCCATCTTCTATGC & 194 \\
Rplpo & GGGCATCACCACGAAAATCTC & CTGCCGTTGTCAAACACCT & 168 \\
\hline
\end{tabular}


A
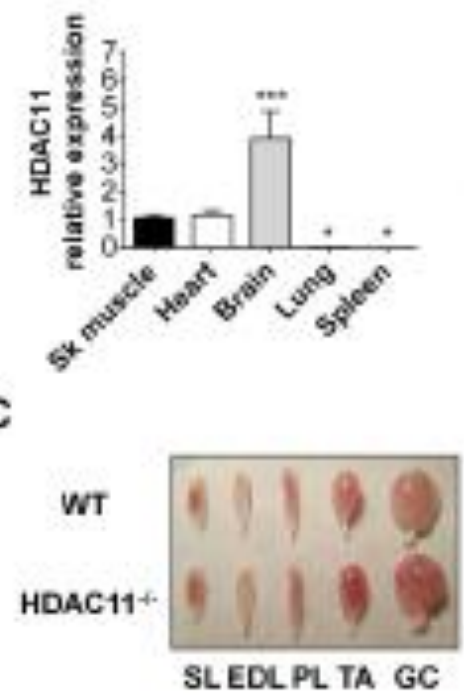

B
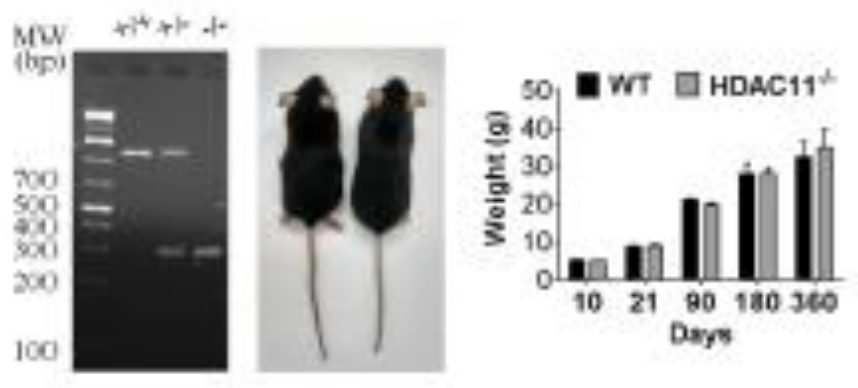

D
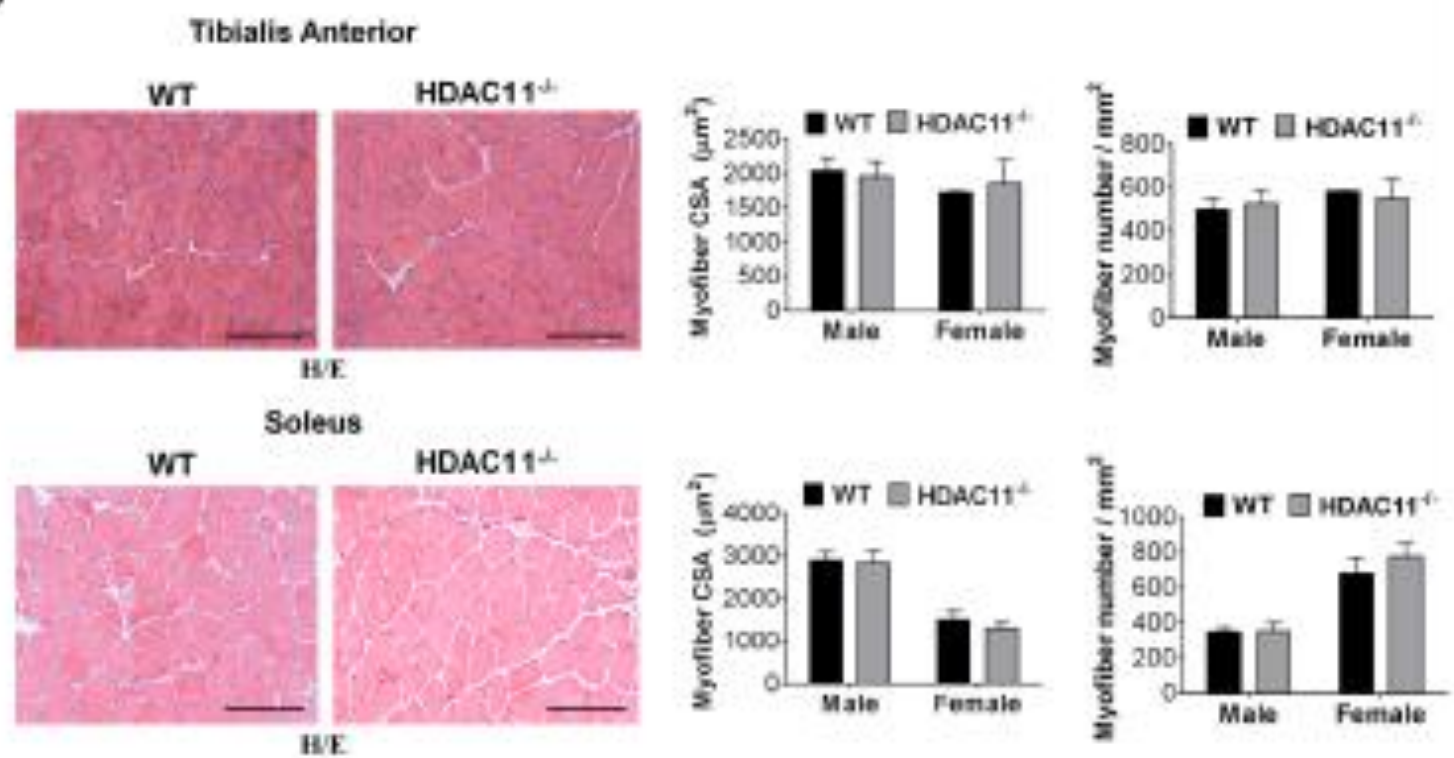

Fig 1. 
A

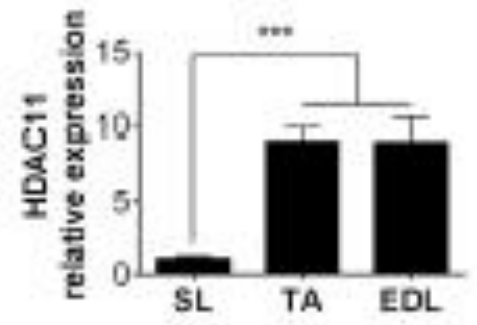

B

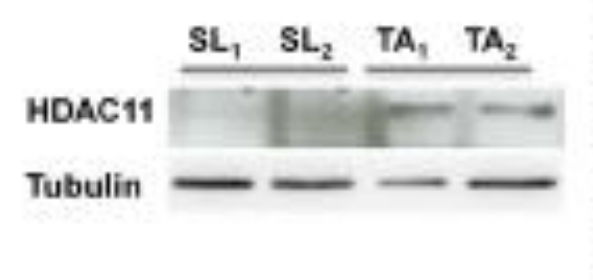

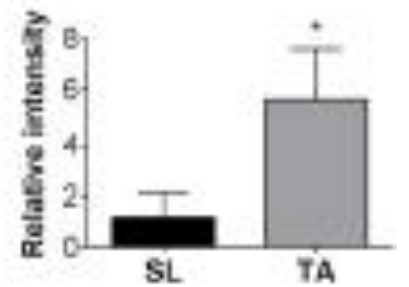

C

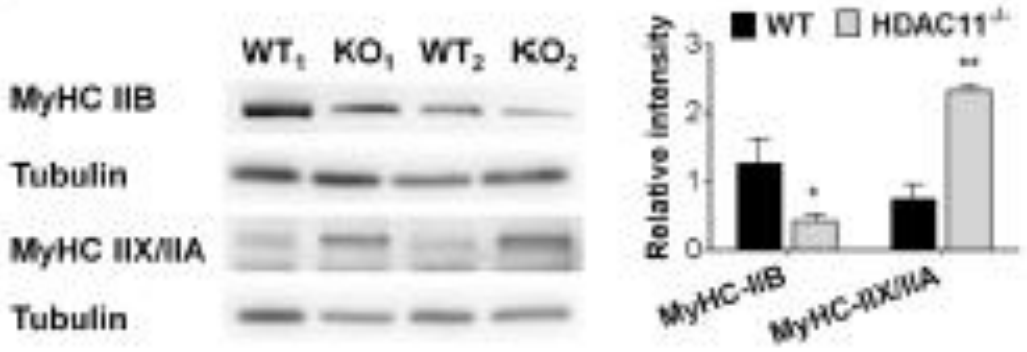

D
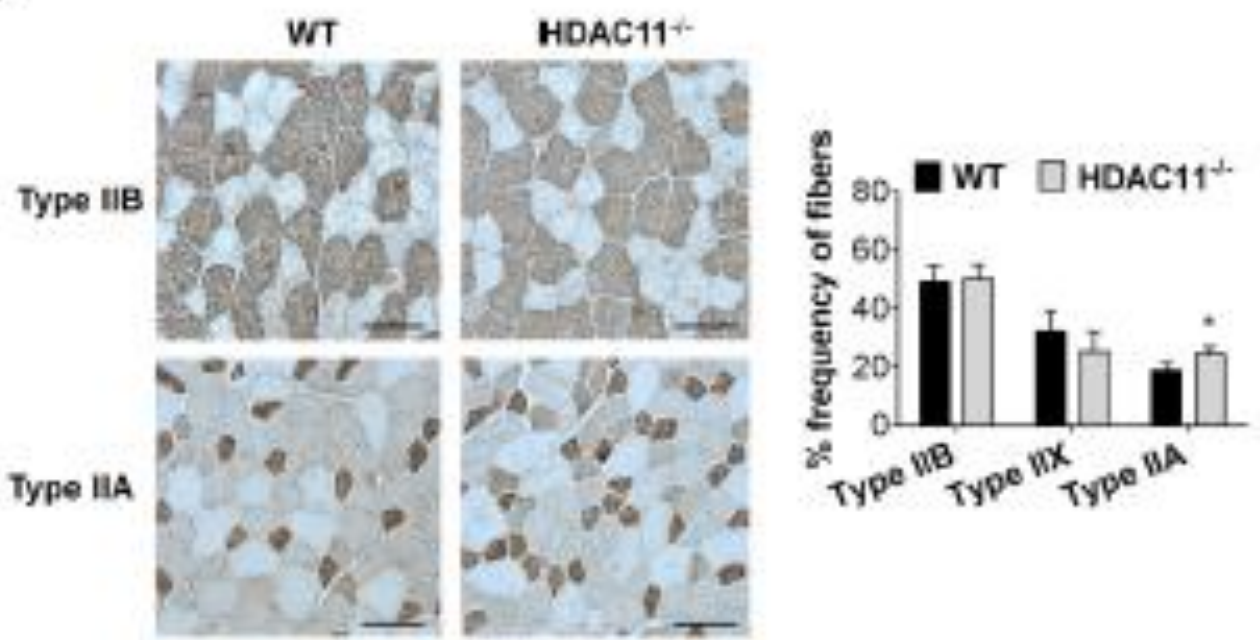

Fig 2 

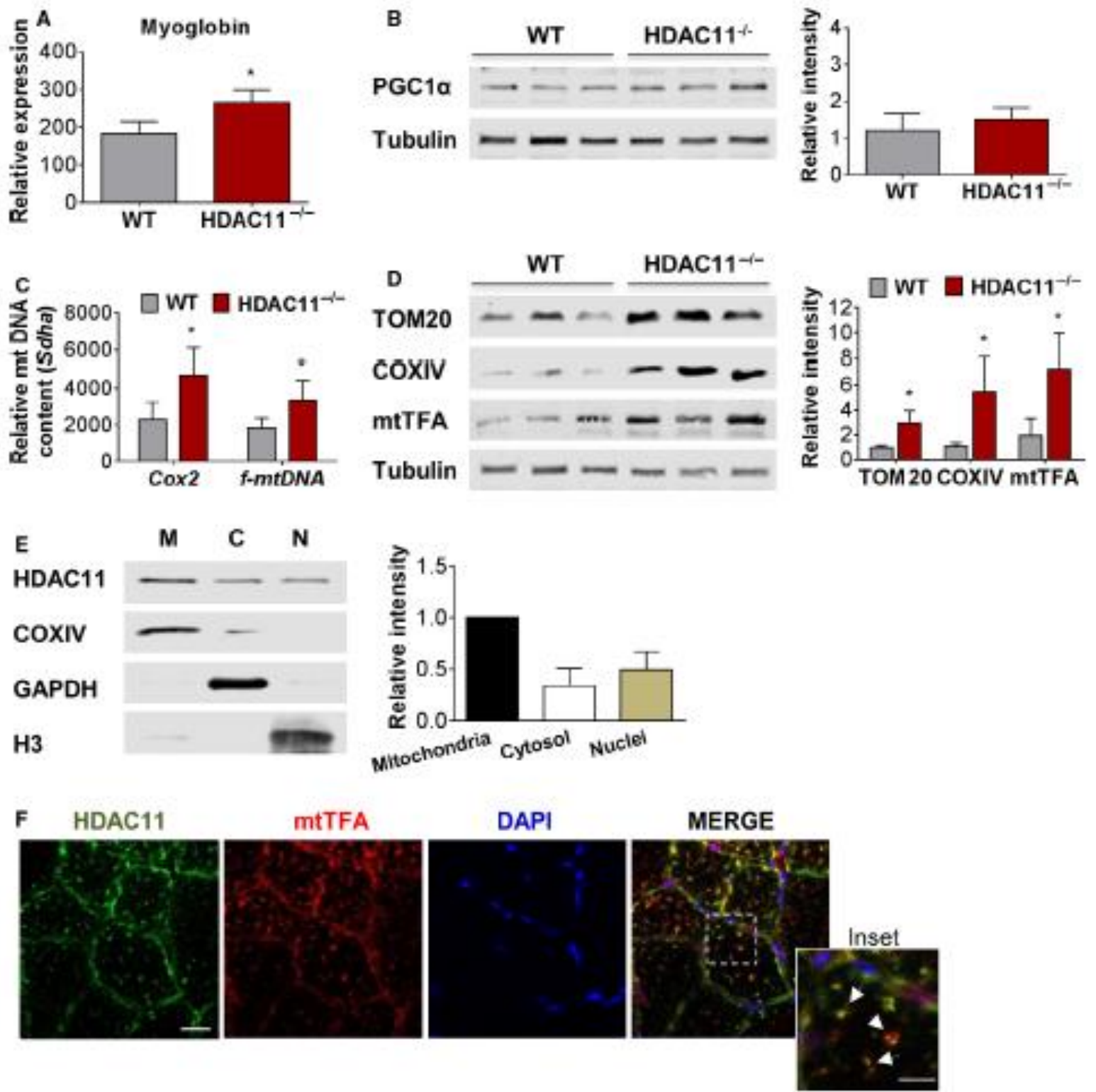

Fig 3 
A

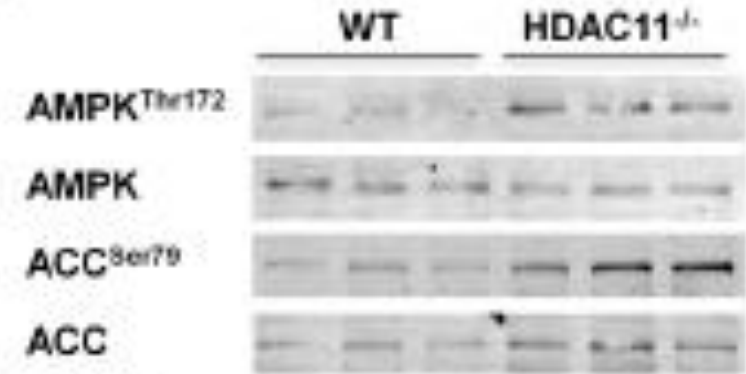

Tubulin

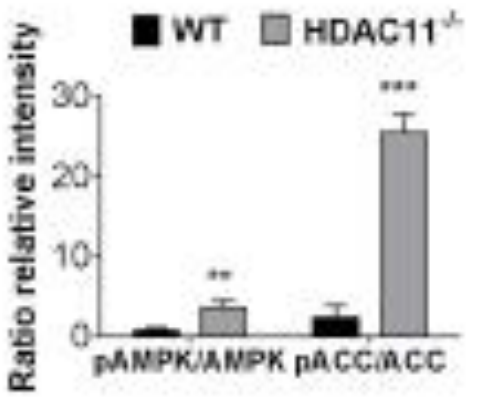

B
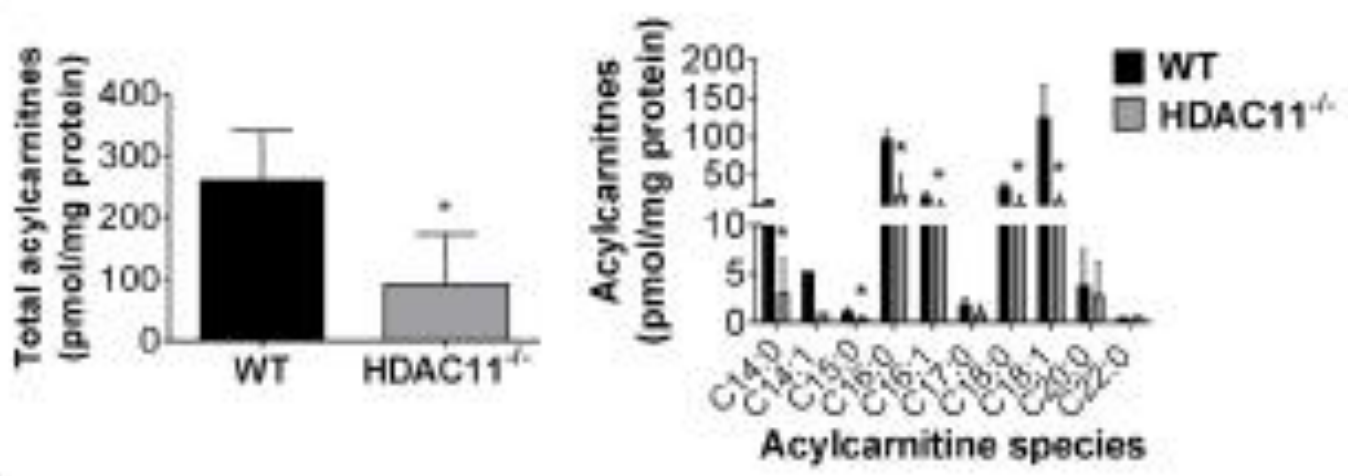

C
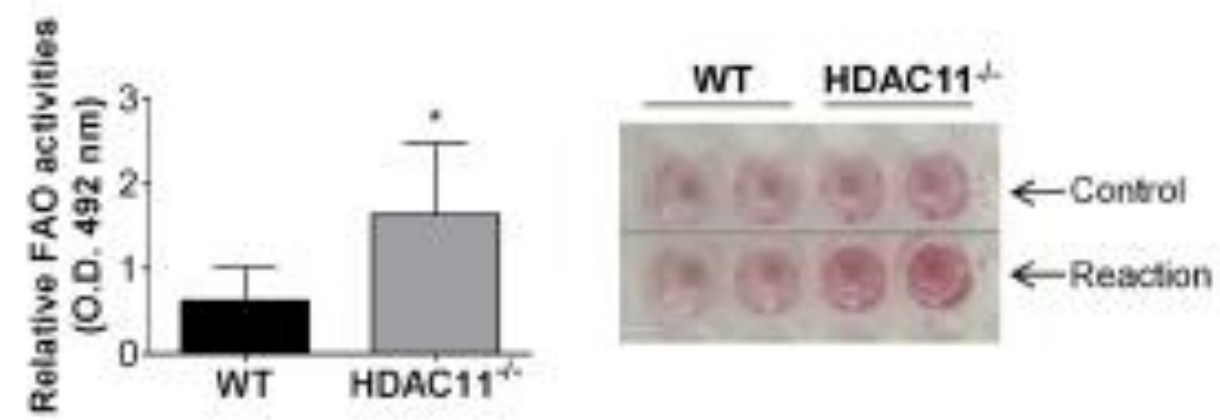

Fig 4 
A
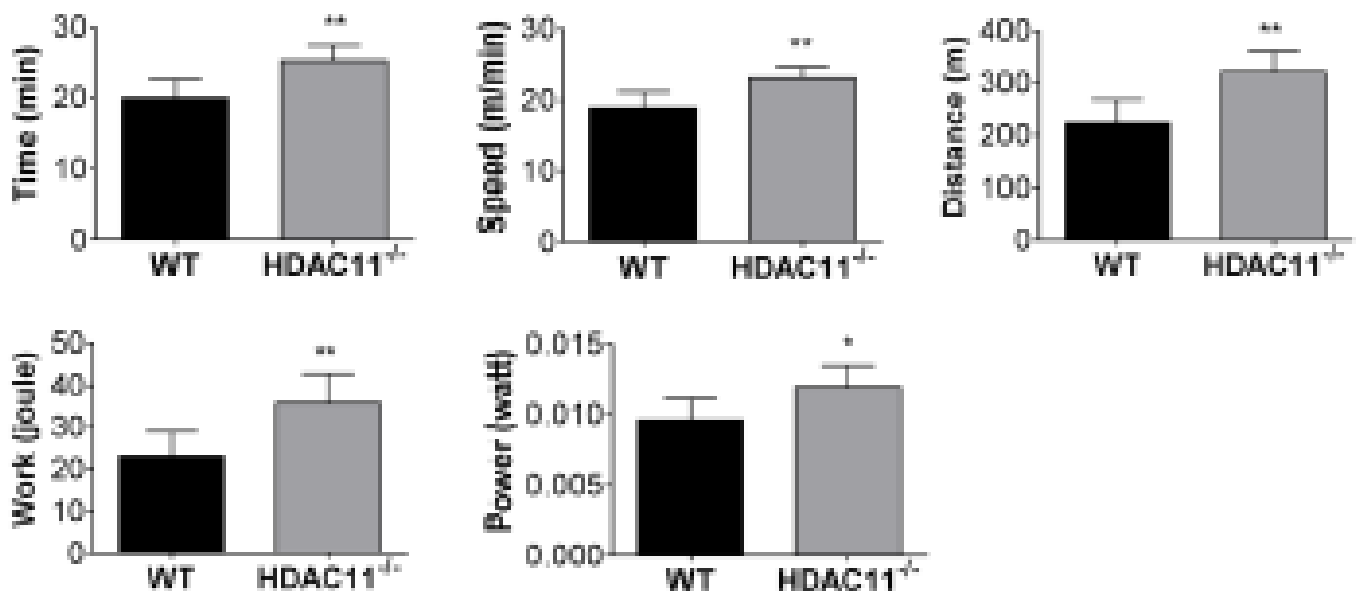

B

C
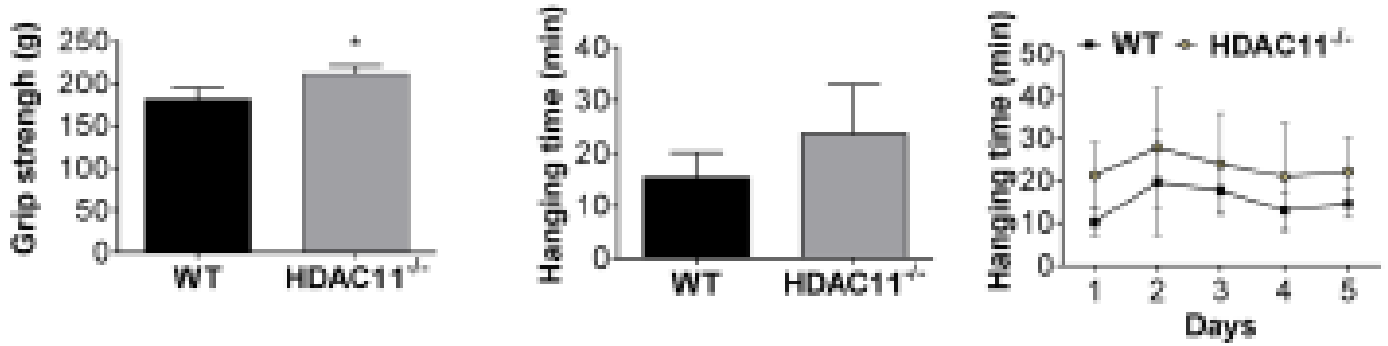

Fig 5 
HDAC11 in skeletal muscle tissue

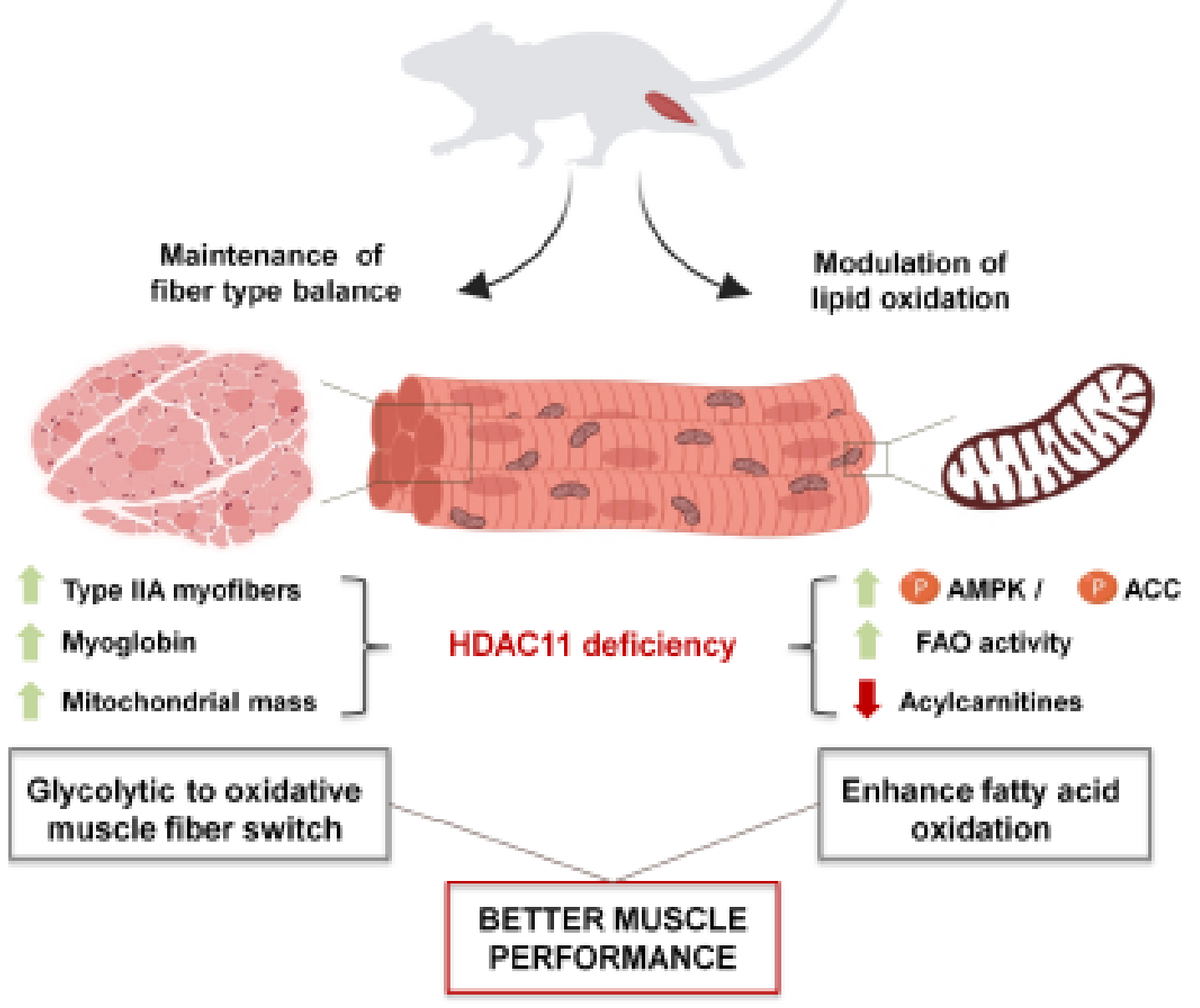

Fig 6 
A
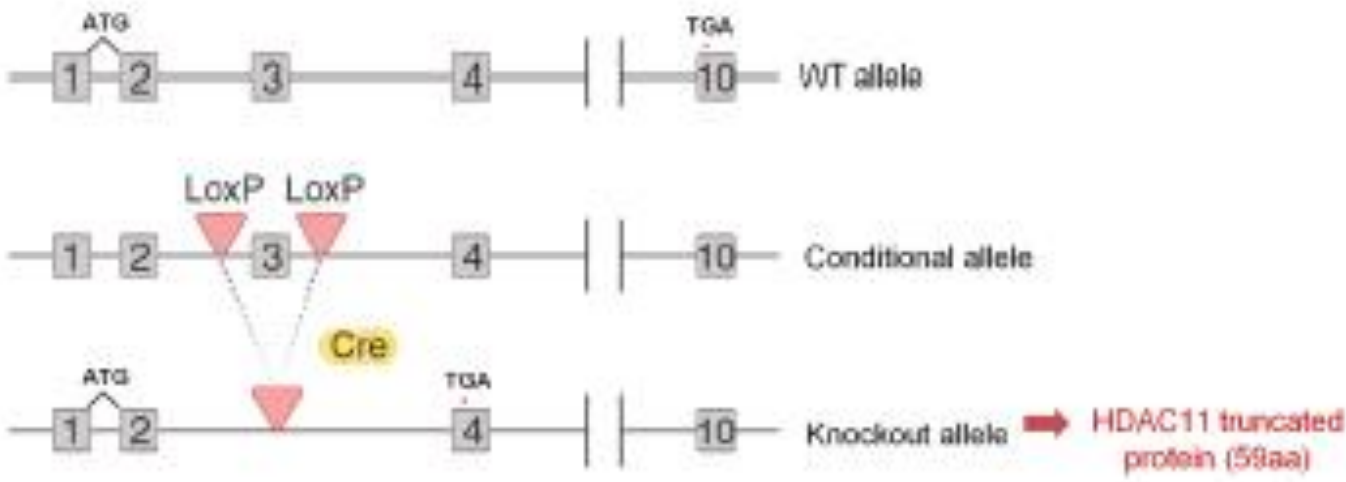

B

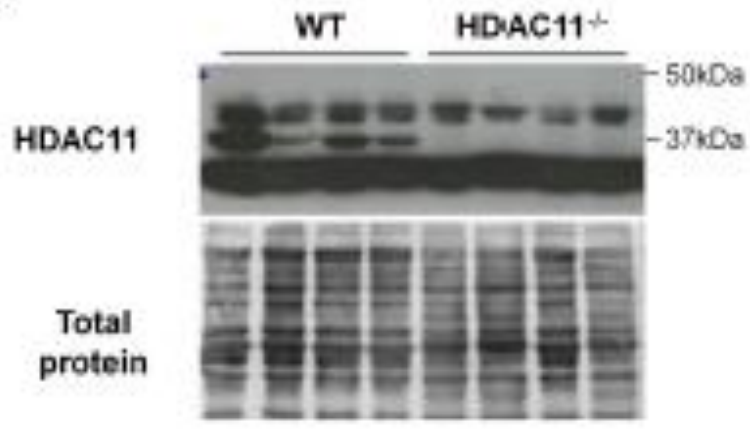

C

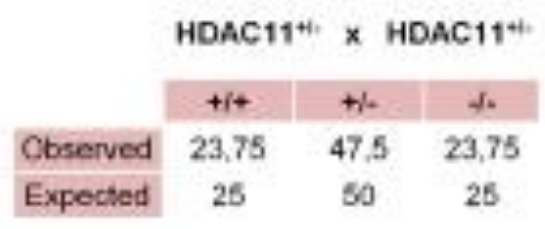

Supplemental Fig 1

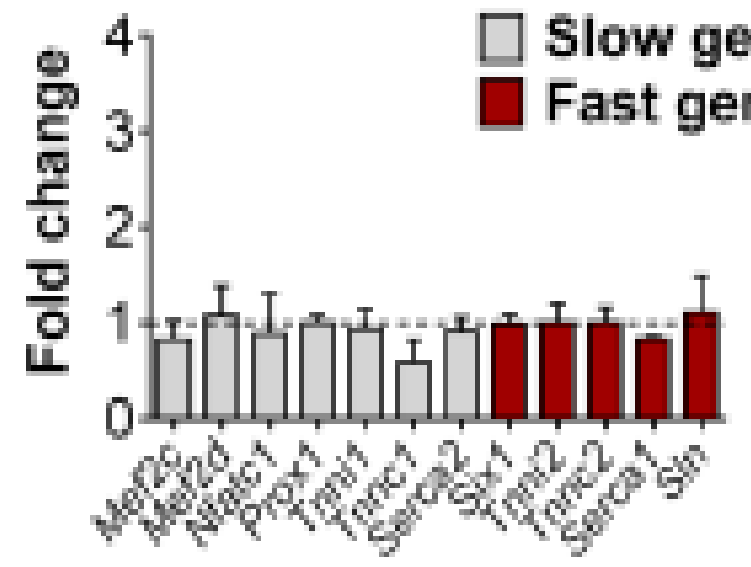

Supplemental Fig 2 